JOURNAL OF

SYMPLECTIC GEOMETRY

Volume 6, Number 4, 407-460, 2008

\title{
ISOTOPIES OF LEGENDRIAN 1-KNOTS AND LEGENDRIAN 2-TORI
}

\author{
ToBias EkHOLM AND TAMÁs KÁlmán
}

\begin{abstract}
We construct a Legendrian 2-torus in the 1-jet space of $S^{1} \times \mathbb{R}$ (or of $\mathbb{R}^{2}$ ) from a loop of Legendrian knots in the 1-jet space of $\mathbb{R}$. The differential graded algebra (DGA) for the Legendrian contact homology of the torus is explicitly computed in terms of the DGA of the knot and the monodromy operator of the loop. The contact homology of the torus is shown to depend only on the chain homotopy type of the monodromy operator. The construction leads to many new examples of Legendrian knotted tori. In particular, it allows us to construct a Legendrian torus with DGA which does not admit any augmentation (linearization) but which still has non-trivial homology, as well as two Legendrian tori with isomorphic linearized contact homologies but with distinct contact homologies.
\end{abstract}

\section{Introduction}

Let $M$ be a smooth $n$-manifold and consider the 1 -jet space $J^{1}(M)=$ $T^{*} M \times \mathbb{R}$ with coordinates $(x, y, z)$, where $x \in M, y \in T_{x}^{*} M$, and $z \in \mathbb{R}$. The 1 -form $\alpha=d z-\sum_{j} y_{j} d x_{j}$ is a contact 1 -form and $\xi=\operatorname{ker}(\alpha)$ is the standard contact structure on $J^{1}(M)$. The Reeb vector field of a contact form $\beta$ is the unique vector field $R_{\beta}$ which satisfies $d \beta\left(R_{\beta}, \cdot\right)=0$ and $\beta\left(R_{\beta}\right)=0$. Hence, if $\alpha$ is the standard contact form then $R_{\alpha}=\partial_{z}$. An $n$-dimensional submanifold $L \subset J^{1}(M)$ which is everywhere tangent to $\xi$ is called Legendrian. The isotopy problem for Legendrian submanifolds is the problem of distinguishing the path components of the space of Legendrian submanifolds. It is a very rich and interesting problem; see e.g. $[\mathbf{3}, \mathbf{6}, \mathbf{1 0}, \mathbf{1 6}, \mathbf{1 7}]$. In $[\mathbf{1 5}]$ the related problem of detecting non-contractible loops in the space of Legendrian 1-submanifolds of $J^{1}(\mathbb{R})$ was studied. In this paper we relate 
the latter problem to the former problem for Legendrian tori in $J^{1}\left(\mathbb{R} \times S^{1}\right)$ (and in $J^{1}\left(\mathbb{R}^{2}\right)$ ).

The introduction of Legendrian contact homology (see $[\mathbf{3}, \mathbf{1 1}]$ ), led to major breakthroughs in the study of the Legendrian isotopy problem. The contact homology of a Legendrian submanifold $L \subset J^{1}(M)$ is the homology of a differential graded algebra (DGA) $\mathcal{A}(L)$ associated to $L$. Here $\mathcal{A}(L)$ is the (non-commutative) unital DGA freely generated by the Reeb chords of $L$ (flow lines of the Reeb vector field starting and ending on $L$ ) graded by their Conley-Zehnder indices. (For simplicity, throughout this paper we will work with $\mathbb{Z}_{2}$-coefficients so that $\mathcal{A}(L)$ is an algebra over a $\mathbb{Z}_{2}$-group ring, rather than a $\mathbb{Z}$-group ring.) The differential on $\mathcal{A}(L)$ is defined using moduli spaces of boundary-punctured holomorphic disks in the symplectization $J^{1}(M) \times \mathbb{R}$ with Lagrangian boundary condition $L \times \mathbb{R} \subset J^{1}(M) \times \mathbb{R}$ and with certain asymptotic properties near the punctures; see Sections 2.1 and 2.2. For 1-dimensional Legendrian submanifolds of $J^{1}(\mathbb{R})$, the Riemann mapping theorem allows for a purely combinatorial description of contact homology; see $[\mathbf{3}]$. For higher-dimensional Legendrian submanifolds, analytical foundations for contact homology were worked out in [6, 8]. In [4], a more combinatorial description was obtained: Legendrian contact homology in 1-jet spaces was described entirely in terms of Morse theoretic objects called flow trees; see Section 2.3.

Contact homology has certain functorial properties. In particular, each isotopy of Legendrian knots induces a Lagrangian cobordism which in turn induces a morphism of the contact homologies at its endpoints. Such morphisms induced by isotopies were dealt with from a purely combinatorial point of view in $[\mathbf{1 5}]$. (The techniques used in this paper lead to a description of these morphisms in terms of moduli spaces of holomorphic curves in a cobordism; see $[\mathbf{9}]$.)

In order to state the main theorem of the paper we first describe the underlying geometric construction. Let $L \subset J^{1}(M)$ be a Legendrian submanifold which is in sufficiently general position with respect to the projection $\Pi_{\mathrm{F}}: J^{1}(M) \rightarrow J^{0}(M)$. The image $\Pi_{\mathrm{F}}(L)$ is the front of $L$ and determines $L$. If $\gamma(t), t \in S^{1}$, is a 1-parameter family of Legendrian submanifolds, starting and ending at $L$, then $\left(\Pi_{\mathrm{F}}(\gamma(t)), t\right) \subset J^{0}\left(M \times S^{1}\right)$ is the front of a Legendrian embedding $L \times S^{1} \rightarrow J^{1}\left(M \times S^{1}\right)$. We call this Legendrian embedding the trace of the isotopy and denote the corresponding Legendrian submanifold by $\Sigma_{\gamma}(L)$. When $M=\mathbb{R}$, consider an embedding $S^{1} \times \mathbb{R} \rightarrow \mathbb{R}^{2}$ obtained by identifying $S^{1} \times \mathbb{R}$ with a small tubular neighborhood of the unit circle. Using this embedding we may consider $\Sigma_{\gamma}(L) \subset J^{1}\left(S^{1} \times \mathbb{R}\right) \subset J^{1}\left(\mathbb{R}^{2}\right)$.

See Figure 1 for the front of $\Sigma_{\gamma}(L)$ in the simplest case when $L$ is the standard Legendrian unknot and $\gamma$ is the constant isotopy. Note that if $\gamma$ is a constant isotopy then for each Reeb chord of $L, \Sigma_{\gamma}(L)$ has an $S^{1}$ family of Reeb chords. The Reeb chord families are analogous to critical 
submanifolds of a Morse-Bott function, and after a small perturbation each Reeb chord family of $\Sigma_{\gamma}(L)$ contributes two Reeb chords of Conley-Zehnder indices equal to, respectively, one larger than that of the corresponding chord of $L$. The following result shows that DGAs of general isotopy traces admit presentations with generators similar to the Reeb chords of traces of constant isotopies and gives the differential in terms of the contact homology morphism induced by the isotopy.

Theorem 1.1. Let $\gamma(t), t \in S^{1}$, be a loop of Legendrian submanifolds of $J^{1}(\mathbb{R})$ starting and ending at $L \subset J^{1}(\mathbb{R})$. Let $\mathcal{A}=\mathbb{Z}_{2}\left[H_{1}(L)\right]\left\langle c_{1}, \ldots, c_{r}\right\rangle$ be the DGA of $L$, let $\left|c_{j}\right|$ denote the degree of the generator $c_{j}$, and let $\phi: \mathcal{A} \rightarrow \mathcal{A}$ be the monodromy operator associated to $\gamma$. Then the DGA of $\Sigma_{\gamma}(L)$ as a Legendrian submanifold of $J^{1}\left(S^{1} \times \mathbb{R}\right)$ or of $J^{1}\left(\mathbb{R}^{2}\right)$ is stable tame isomorphic to the algebra $(\hat{\mathcal{A}}, \Delta)$, where

$$
\hat{\mathcal{A}}=\mathbb{Z}_{2}\left[H_{1}\left(L \times S^{1}\right)\right]\left\langle c_{1}, \ldots, c_{r}, \hat{c}_{1}, \ldots, \hat{c}_{r}\right\rangle,
$$

with $\left|\hat{c}_{j}\right|=\left|c_{j}\right|+1$ for all $j$, and where

$$
\begin{aligned}
& \Delta\left(c_{j}\right)=\partial c_{j}, \\
& \Delta\left(\hat{c}_{j}\right)=c_{j}+\phi\left(c_{j}\right)+\Gamma_{\phi}\left(\partial c_{j}\right),
\end{aligned}
$$

with $\Gamma_{\phi}: \mathcal{A} \rightarrow \hat{\mathcal{A}}$ denoting the degree 1 derivation defined by $\Gamma\left(c_{s}\right)=\hat{c}_{s}$ and extended to all of $\mathcal{A}$ by

$$
\Gamma_{\phi}(\alpha \beta)=\Gamma_{\phi}(\alpha) \phi(\beta)+\alpha \Gamma_{\phi}(\beta) .
$$

Theorem 1.1 is proved in Section 7. Using it, we provide examples establishing the following.

Theorem 1.2. Let $Y$ denote $J^{1}\left(S^{1} \times \mathbb{R}\right)$ or $J^{1}\left(\mathbb{R}^{2}\right)$ equipped with its standard contact form.

(a) There exists a Legendrian torus in $Y$ with DGA which has no augmentation but which has non-zero homology.

(b) There exists a Legendrian torus in $Y$ with linearized contact homology isomorphic to the linearized contact homology of the standard torus (the standard torus is the trace of the constant isotopy of the unknot) but with full contact homology different from that of the standard torus.

Theorem 1.2 is proved in Section 8; see [18] for similar results for 1-dimensional Legendrian submanifolds. The Legendrian tori in (a) and (b) are the traces of isotopies of Legendrian $(3,2)$ and $(7,2)$ torus knots, respectively, that were studied in $[\mathbf{1 5}]$. We show they have properties as claimed by computing their contact homology algebras in degree 0 . 
The paper is organized as follows. In Section 2 we recall the definition of contact homology and describe how to compute it in 1-jet spaces using flow trees. In Section 3 we discuss certain slight generalizations of contact homology described in terms of perturbed flow trees. In Section 4 we explain how the differential $\partial$ of the DGA $\mathcal{A}$ of the trace of the constant isotopy can be computed in terms of perturbed flow trees which arise from a geometric perturbation of the Legendrian submanifold. This computation is however not explicit enough to yield a closed formula for $\partial$. In Section 5 we design an abstract perturbation for the trace of the constant isotopy which yields a closed formula for another differential $\partial^{\prime}$ on the DGA $\mathcal{A}$. By a result from Section 3 , the DGAs $(\mathcal{A}, \partial)$ and $\left(\mathcal{A}, \partial^{\prime}\right)$ are tame isomorphic. In Section 6 we discuss how to decompose an isotopy into simple pieces and how to compute the DGAs of the traces of such simple pieces. Concatenation of the pieces then yields the DGA for the full isotopy. However, the concatenation gives a DGA with an enormous number of generators. In Section 7 we show, using a purely algebraic argument, that the DGA with the large number of generators is stable tame isomorphic to the DGA in the formulation of Theorem 1.1. We also give an algebraic proof of fact that the contact homology of the trace of an isotopy depends only on the chain homotopy class of the morphism of the isotopy. In Section 8 we study examples needed to establish Theorem 1.2.

\section{Background}

In this section we give a brief description of Legendrian contact homology and how to compute it for Legendrian submanifolds in 1-jet spaces. For details we refer to $[\mathbf{4}, \mathbf{6}, \mathbf{8}]$. More precisely, the DGA associated to a Legendrian submanifold is described in Sections 2.1 and 2.2. In Section 2.3 we define flow trees and describe the relation between rigid flow trees and rigid holomorphic disks.

2.1. Holomorphic disks. Let $M$ be a smooth $n$-manifold, endow $J^{1}(M)=$ $T^{*} M \times \mathbb{R}$ with its standard contact form, and let $z$ be a coordinate in the $\mathbb{R}$-direction. Let $L$ be a closed Legendrian submanifold . We assume that $L$ is sufficiently generic so that the Lagrangian projection $\Pi_{\mathbb{C}}: J^{1}(M) \rightarrow T^{*} M$ restricted to $L$ has only transverse double points. If $c$ is a double point of $\Pi_{\mathbb{C}}(L)$ then we write $\Pi_{\mathbb{C}}^{-1}(c) \cap L=\left\{c^{+}, c^{-}\right\}$, where $z\left(c^{+}\right)>z\left(c^{-}\right)$. (Since the Reeb field of the standard contact form $d z-y d x$ is $\partial_{z}$, there exists a 1-1 correspondence between double points of $\Pi_{\mathbb{C}}(L)$ and Reeb chords on $L$, and we will use these two notions interchangeably.)

Let $D_{m+k}$ be the unit disk $D$ in the complex plane $\mathbb{C}$ with punctures $x_{1}, \ldots, x_{m}, y_{1}, \ldots, y_{k}$ on the boundary, and let $J$ be an almost complex structure on $T^{*} M$ which is tamed by the standard symplectic form $\omega=d x \wedge d y$ on $T^{*} M$. 
Definition 2.1. A $J$-holomorphic disk with positive punctures $p_{1}, \ldots, p_{m}$ and negative punctures $q_{1}, \ldots, q_{k}$ and with boundary on $L$ is a map $u: D_{m+k} \rightarrow T^{*} M$ with the following properties.

- $\bar{\partial}_{J} u=d u+J \circ d u \circ i=0$ (where $i$ is the complex structure on the complex plane).

- The restriction $u \mid \partial D_{m+k}$ has a continuous lift $\tilde{u}: \partial D_{m+k} \rightarrow L \subset$ $J^{1}(M)$.

- $\lim _{\zeta \rightarrow x_{j}} u(\zeta)=p_{j}$ and $\lim _{\zeta \rightarrow x_{j} \pm} \tilde{u}(\zeta)=p_{j}^{ \pm}$, where $\lim _{\zeta \rightarrow x_{j}}+$ means that $\zeta$ approaches $x_{j}$ from the region in $\partial D_{m+k}$ in the counterclockwise direction as seen from $x_{j}$ and $\lim _{\zeta \rightarrow x_{j}-}$ means it approaches $x_{j}$ from the region in the clockwise direction.

- $\lim _{\zeta \rightarrow y_{j}} u(\zeta)=q_{j}$ and $\lim _{\zeta \rightarrow y_{j} \pm} \tilde{u}(\zeta)=q_{j}^{\mp}$.

In this paper we will restrict attention to holomorphic disks with exactly one positive puncture. The moduli space of holomorphic disks with boundary on $L$ has certain compactness properties: a version of Gromov's compactness theorem holds; see $[\mathbf{2}, \mathbf{5}, \mathbf{8}]$. Since the linearized $\bar{\partial}_{J}$-operator is elliptic and since the double points of $\Pi_{\mathbb{C}}(L)$ are transverse, the linearization of the equation which defines the moduli space of $J$-holomorphic disks is a Fredholm operator and its index determines the expected dimension of the moduli space. Details of the computation of the Fredholm index can be found in $[\mathbf{5}, \mathbf{8}]$. Here we simply state the result. Pick for each Reeb chord $c$ of $L$ a capping path $\gamma_{c}$ in $L$ connecting $c^{+}$to $c^{-}$. Define $\Gamma_{c}$ to be the path of Lagrangian subspaces $\Pi_{\mathbb{C}}\left(T_{\gamma_{c}} L\right)$ closed up by a positive rotation. Define

$$
|c|=\mu\left(\Gamma_{c}\right)-1,
$$

where $\mu$ is the Maslov index. Given any boundary condition of a holomorphic disk with positive puncture at $a$ and negative punctures at $b_{1}, \ldots, b_{k}$, we can close it up to a loop by adding appropriately oriented capping paths at the Reeb chords. Let $A \in H_{1}(L ; \mathbb{Z})$ denote the homology class of the closed-up loop and let $\mathcal{M}_{A}\left(a ; b_{1}, \ldots, b_{k}\right)$ denote the moduli space of holomorphic disks with positive puncture $a$, negative punctures at $b_{1}, \ldots, b_{k}$, and boundary condition inducing the homology class $A$. Then

$$
\operatorname{dim}\left(\mathcal{M}_{A}\left(a ; b_{1}, \ldots, b_{k}\right)\right)=|a|-\sum_{j=1}^{k}\left|b_{k}\right|+\mu\left(\Gamma_{A}\right)-1,
$$

where $\Gamma_{A}$ is the path of Lagrangian subspaces along a loop in $L$ representing $A$.

2.2. Legendrian contact homology. Let $L \subset J^{1}(M)$ be a Legendrian submanifold. The DGA $\mathcal{A}(L)$ of $L$ is the free algebra over the $\mathbb{Z}_{2}$-group ring of $H_{1}(L ; \mathbb{Z})$ generated by the Reeb chords of $L$,

$$
\mathcal{A}(L)=\mathbb{Z}_{2}\left[H_{1}(L ; \mathbb{Z})\right]\left\langle c_{1}, \ldots, c_{m}\right\rangle .
$$


The differential $\partial: \mathcal{A}(L) \rightarrow \mathcal{A}(L)$ counts rigid holomorphic disks. It is defined to be linear over the algebra coefficients and to satisfy Leibniz rule and is thus determined by its action on generators. If $a$ is a generator then

$$
\partial a=\sum_{\operatorname{dim}\left(\mathcal{M}_{A}\left(a ; b_{1}, \ldots, b_{k}\right)\right)=0}\left|\mathcal{M}_{A}\left(a ; b_{1}, \ldots, b_{k}\right)\right| A b_{1} \cdots b_{k},
$$

where $|\mathcal{M}|$ denotes the modulo 2 number of points in the compact 0-manifold $\mathcal{M}$. For this definition to make sense we require that $L$ is generic with respect to holomorphic disks so that all moduli spaces of holomorphic disks of dimension $\leq 1$ are transversely cut out. It is shown in $[\mathbf{5}, \mathbf{7}]$ how to achieve such transversality by perturbing $L$. In these papers it is also shown that $\partial: \mathcal{A}(L) \rightarrow \mathcal{A}(L)$ is a differential (i.e., $\partial^{2}=0$ ) and that the contact homology $\operatorname{ker}(\partial) / \operatorname{im}(\partial)$ is invariant under Legendrian isotopies.

Remark 2.2. In the case that $L$ is a spin manifold and $M$ is orientable, one can lift the DGA over $\mathbb{Z}_{2}\left[H_{1}(L ; \mathbb{Z})\right]$ described above to a DGA over $\mathbb{Z}\left[H_{1}(L ; \mathbb{Z})\right]$. In this paper we will, as mentioned in Section 1 , concentrate on the $\mathbb{Z}_{2}$-case. This is mainly for simplicity; the results of the paper have straightforward generalizations to the more general setting of $\mathbb{Z}$-coefficients.

2.3. Flow trees. We refer to $[4]$ for the detailed definitions of flow trees and only sketch the main points here. Let $L \subset J^{1}(M)$ be a Legendrian submanifold. Locally around points outside a codimension 1 subset of $M$, the image of $L$ under the front projection $\Pi_{\mathrm{F}}: J^{1}(M) \rightarrow J^{0}(M)=M \times \mathbb{R}$, can be described as the graphs of a finite number of functions on $M$. We call such functions local functions of $L$. If $M$ is equipped with a Riemannian metric then these local functions define local gradients. We say that a curve $\gamma(t)$ in $M$ is a flow line of $L$ if it satisfies the differential equation

$$
\dot{\gamma}(t)=-\nabla\left(f_{1}-f_{2}\right)(\gamma(t)),
$$

where $f_{1}$ and $f_{2}$ are local functions of $L$. Any flow line has a natural 1-jet lift, which consists of two curves in $L$ lying over $\gamma$. These curves are naturally oriented by the lifts of the vectors $-\nabla\left(f_{1}-f_{2}\right)$ and $-\nabla\left(f_{2}-f_{1}\right)$ to the sheets corresponding to $f_{1}$ and $f_{2}$, respectively. Projecting the 1 -jet lift to the cotangent bundle we get the cotangent lift.

Definition 2.3. A flow tree of $L \subset J^{1}(M)$ is a continuous map $\phi: \Gamma \rightarrow M$, where $\Gamma$ is a tree which satisfies the following conditions.

(a) If $e$ is an edge of $\Gamma$ then $\phi: e \rightarrow M$ is an injective parameterization of a flow line of $L$.

(b) Let $v$ be a $k$-valent vertex with cyclically ordered adjacent edges $e_{1}, \ldots, e_{k}$. Let $\left\{\bar{\phi}_{j}^{1}, \bar{\phi}_{j}^{2}\right\}$ be the cotangent lift corresponding to $e_{j}$, $1 \leq j \leq k$. We require that there exists a pairing of lift components such that for every $1 \leq j \leq k$ (with $k+1 \equiv 1)$

$$
\bar{\phi}_{j}^{2}(v)=\bar{\phi}_{j+1}^{1}(v)=\bar{m} \in \Pi_{\mathbb{C}}(L) \subset T^{*} M,
$$


and such that the flow orientation of $\bar{\phi}_{j}^{2}$ at $\bar{m}$ is directed toward $\bar{m}$ if and only if the flow orientation of $\bar{\phi}_{j+1}^{1}$ at $\bar{m}$ is directed away from $\bar{m}$.

(c) The cotangent lifts of the edges of $\Gamma$ fit together to an oriented curve $\bar{\phi}$ in $\Pi_{\mathbb{C}}(L)$. We require that this curve is closed.

For simpler notation we will often denote flow trees simply by $\Gamma$, suppressing the parameterization map $\phi$ from the notation. We will also write $\bar{\Gamma}$ and $\tilde{\Gamma}$ for the cotangent and the 1-jet lifts of $\Gamma$, respectively. See Figure 1 for examples of flow trees.

We next define punctures of a flow tree $\Gamma$. Let $v$ be a $k$-valent vertex of $\Gamma$ with cyclically ordered edges $e_{1}, \ldots, e_{k}$. Consider two paired cotangent lifts $\bar{\phi}_{j}^{2}$ and $\bar{\phi}_{j+1}^{1}$ and the corresponding 1 -jet lifts $\tilde{\phi}_{j}^{2}$ and $\tilde{\phi}_{j+1}^{1}$ at $v$. If $\tilde{\phi}_{j}^{2}(v) \neq \tilde{\phi}_{j+1}^{1}(v)$ then both must equal Reeb chord endpoints. If this is the case then we say that $v$ contains a puncture after $e_{j}$. It is shown in [4] that any flow tree with a vertex $v$ which contains more than one puncture is a union of flow trees such that every vertex of each one of them contains at most one puncture. Thus we may restrict attention to flow trees with at most one puncture at each vertex and we call such a vertex a puncture of the tree.

Let $p$ be a puncture of a flow tree. Let $\tilde{\phi}^{1}$ and $\tilde{\phi}^{2}$ be the 1 -jet lifts which map to the Reeb chord at $p$, with notation chosen so that $\tilde{\phi}^{1}$ is oriented toward $\tilde{\phi}^{1}(p)$ and $\tilde{\phi}^{2}$ oriented away from $\tilde{\phi}_{2}(p)$. Then we say that $p$ is a positive puncture if

$$
z\left(\tilde{\phi}^{1}(p)\right)<z\left(\tilde{\phi}^{2}(p)\right)
$$
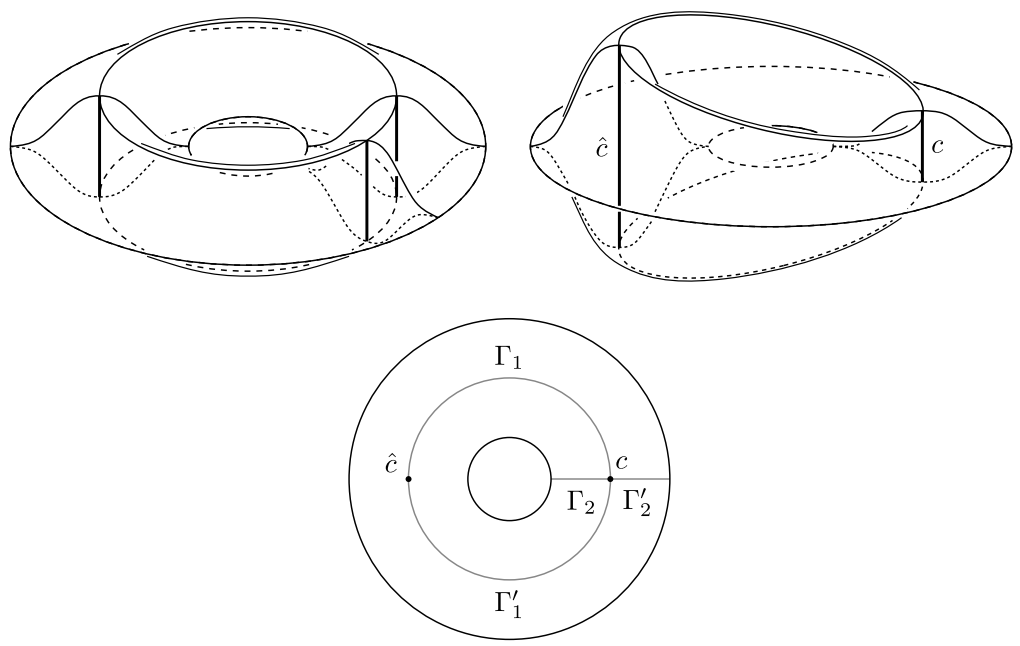

Figure 1. A standard Legendrian torus. 
and we say it is negative if the opposite inequality holds. (Recall that $z$ is the coordinate in the $\mathbb{R}$-direction of $J^{1}(M)=T^{*} M \times \mathbb{R}$.) Using the symplectic area of a flow tree it is not hard to show that every flow tree $\Gamma$ in $M$ has at least one positive puncture. If $q$ is a puncture of a flow tree then $q$ corresponds to some Reeb chord of $L$ which in turn corresponds to a critical point of some local function difference of $L$. If $L$ is generic then the Hessian at this critical point is non-degenerate. Let $I(q)$ denote the index of the critical point of the positive function difference corresponding to the Reeb chord.

As for holomorphic disks there is a simple dimension formula for flow trees which computes the expected dimension of the space of flow trees with 1-jet lift homotopic to that of the given tree. For trees with exactly one positive puncture this formula reads

$$
\operatorname{dim}(\Gamma)=(I(p)-1)-\sum_{q \in Q(\Gamma)}(I(q)-1)+\sum_{r \in R(\Gamma)} \mu(r)-1,
$$

where $p$ is the positive puncture of $\Gamma, Q(\Gamma)$ its set of negative punctures, $R(\Gamma)$ its set of vertices which are not punctures, and $\mu(r)$ is the Maslov content of $r$; see [4, Section 3.1.1].

Example 2.4. In Figure 1 the rigid flow trees of the standard Legendrian torus are shown. The top left picture shows the torus with an $S^{1}$-family of Reeb chords. The top right picture shows the torus after perturbation. Now it has two Reeb chords $\hat{c}$ and $c$ which satisfy $I(\hat{c})=2$ and $I(c)=1$, respectively. The rigid flow lines $\Gamma_{1}$ and $\Gamma_{1}^{\prime}$ connect $\hat{c}$ to $c$ and the rigid flow trees $\Gamma_{2}$ and $\Gamma_{2}^{\prime}$ have their positive puncture at $c$ and an end at the cusp edge. Gluing $\Gamma_{1}$ and $\Gamma_{2}\left(\Gamma_{1}\right.$ and $\left.\Gamma_{2}^{\prime}\right)$ we get a 1-parameter family of trees which end at the broken tree consisting of $\Gamma_{1}^{\prime}$ and $\Gamma_{2}\left(\Gamma_{1}^{\prime}\right.$ and $\left.\Gamma_{2}^{\prime}\right)$.

Viewing the 1-jet lift as a boundary condition for a holomorphic disk, the dimension formula just stated agrees with the dimension formula for holomorphic disks. In fact for rigid disks and trees, more is true as the following theorem shows. (See [4, Theorem 1.1] for a more general version.)

Theorem 2.5. If $L \subset J^{1}(M)$ is a Legendrian submanifold of dimension $\leq 2$ then there is a generic complex structure $J$ on $T^{*} M$ such that there is a 1-1 correspondence between rigid $J$-holomorphic disks with boundary on $L$ and rigid flow trees determined by $L$.

This theorem has the consequence that one may replace holomorphic disks in the definition of Legendrian contact homology differential with flow trees. We will often do so below.

We will sometimes talk about convergence of flow trees. When doing so we employ the following topology on the space of flow trees. Let $\Gamma$ be a flow tree with one positive puncture $p_{0}$. Orient $\Gamma$ by declaring any edge 
starting at $p_{0}$ to be oriented away from $p_{0}$ and by requiring that at each vertex different from $p_{0}$ there is exactly one edge oriented toward it. We will associate a planar domain $\Delta_{\Gamma}$ to $\Gamma$ as follows. For each 1-valent vertex different from $p_{0}$ and for each puncture different from $p_{0}$, pick a half infinite strip of width 1. For each vertex where such edges begin, pick a strip of width which equals the sum of the widths of the edges going out from it and of length agreeing with the natural flow length of the edge coming in to that vertex. Gluing the half strips to the strip, we obtain a half strip domain of finite width with slits toward $+\infty$. (Here we consider the half strip of a negative puncture at a vertex of valence $\geq 2$ as outgoing from that vertex.) Continuing inductively in this way, we get a strip domain with slits toward $+\infty$ and where $-\infty$ corresponds to $p_{0}$. This domain $\Delta_{\Gamma}$ is determined up to over-all translation of the slits. Thus the conformal structure on $\Delta_{\Gamma}$ thought of as a disk with boundary punctures is uniquely determined by $\Gamma$.

Note that the cotangent lift of $\Gamma$ is naturally defined as a map from the boundary $\partial \Delta_{\Gamma}$ to $\Pi_{\mathbb{C}}(L)$. Consider the bundle of mapping spaces of maps from the boundary of a punctured disk into $\Pi_{\mathbb{C}}(L)$ over the space of conformal structures on the disk with $m$ punctures, endowed with the $C^{0}$-topology. We topologize the space of flow trees by giving it the subspace topology with respect to this topology on the bundle of mapping spaces.

\section{Generalizations of Legendrian contact homology}

In this section, we generalize Legendrian contact homology slightly. The first generalization concerns a special kind of non-closed Legendrian submanifolds which can be concatenated in a natural way, see Section 3.2. The concatenation property will be important in the proof of Theorem 1.1 where isotopies are cut into small pieces which are studied separately and then concatenated. The second generalization (see Sections 3.3 and 3.4) concerns stabilization of Legendrian submanifolds. It is more elaborate than the first and is inspired by Morse-Bott techniques for contact homology (see [1]), as well as so-called abstract perturbations (see [12-14]). This generalization will be used in the computation of the contact homology corresponding to a small piece of an isotopy.

3.1. Holomorphic disks and flow trees of 1-dimensional Legendrian submanifolds. Let $L \subset J^{1}(\mathbb{R})$ be a Legendrian submanifold. Using the Riemann mapping theorem, moduli spaces of holomorphic disks with boundary on $L$ can be understood geometrically as follows. The Reeb chords of the diagram correspond to double point of the Lagrangian projection $\Pi_{\mathbb{C}}: J^{1}(\mathbb{R}) \rightarrow T^{*} \mathbb{R} \approx \mathbb{C}$. Holomorphic disks correspond to ordinary holomorphic disks in $\mathbb{C}$ with boundary on the knot diagram $\Pi_{\mathbb{C}}(L)$. The punctures are located at double points of the diagram where the holomorphic disks have corners. A puncture is positive (negative) if, in terms 
of the orientation of the boundary of the holomorphic disk, the boundary component oriented toward (away from) the corner maps to the lower strand of the diagram at the double point and the boundary component oriented away from (toward) the corner maps to the upper strand.

Lemma 3.1. Let $\mathcal{F}$ denote the space of flow trees with one positive puncture determined by $L$, let $\mathcal{M}$ denote the moduli space of all holomorphic disks with one positive puncture determined by $L$, and let $\mathcal{M}^{\prime} \subset \mathcal{M}$ denote the subspace of all disks without interior branch points. Then the spaces $\mathcal{F}$ and $\mathcal{M}^{\prime}$ are homeomorphic and the subset $\mathcal{M}^{\prime} \subset \mathcal{M}$ is closed and has a natural compactification consisting of broken disks from $\mathcal{M}^{\prime}$.

Proof. Let $\Gamma$ be a tree. Joining corresponding points on the cotangent lift of $\Gamma$ by straight lines in the fibers of $T^{*} \mathbb{R}$, we obtain a map of a disk with branch points on the boundary. By the Riemann mapping theorem it admits a holomorphic parameterization. Conversely, any holomorphic disk without interior branch points gives flow lines joined at branch points. That is, a flow tree with higher valence vertices corresponding to branch points. Thus there is a natural bijection between $\mathcal{F}$ and $\mathcal{M}$ as sets. We then need only show that the map $\mathcal{M} \rightarrow \mathcal{F}$ is continuous and open. This however is straightforward: a holomorphic disk is determined by the location of its branch points, varying these give an open subset in the space of trees. Finally, it is clear that the subset of the compactified moduli space of holomorphic disks which consists of (broken) disks without interior branch points is closed. Being a closed subset of a compact space it is compact as well.

Corollary 3.2. The space of flow trees is a manifold with boundary with corners. In particular, the boundary of a component of the space of trees consists of broken trees.

Proof. This holds for the compactified moduli space of holomorphic disks. The corollary thus follows from Lemma 3.1.

Remark 3.3. In order to see the relation between $\mathcal{M}$ and $\mathcal{M}^{\prime}$, note that any disk with interior branch points can be connected to a disk of the same dimension with all its branch points on the boundary by pushing the branch points. For example, a second-order branch point which is pushed to the boundary becomes a third-order boundary branch point which splits into two ordinary boundary branch points. A local model can be obtained as follows. Consider the map $f(z)=z^{2}$ with domain $\Omega$ bounded by the curve $\{2 x y=-\epsilon, x>0\}, \epsilon>0$, and containing the coordinate axes. Note that $f$ maps $\partial \Omega$ to the line $y=-\epsilon$ and that it has a branch point at 0 . To see what happens as the branch point approaches the boundary we let $\epsilon \rightarrow 0$. In the limit $f$ becomes a map $f_{0}$ from the complement of the open fourth quadrant of the complex plane. Consider the map $z(w)=w^{3 / 2}$, taking the upper half plane to the domain of $f_{0}$. Then the map $f_{0}(z(w))$ looks like $w \mapsto w^{3}$ near 
the origin and the 2-parameter family continues as $w \mapsto \delta_{1}+w\left(w^{2}-\delta_{2}\right)$ for $\delta_{1}, \delta_{2} \in \mathbb{R}, \delta_{2}>0$.

3.2. Legendrian submanifolds with standard ends. Let $L \subset J^{1}\left(\mathbb{R}^{n}\right)$ be a Legendrian submanifold parameterized by $\gamma(p)=(x(p), y(p), z(p)) \in$ $J^{1}\left(\mathbb{R}^{n}\right)$. For $\kappa>0$, consider the Legendrian submanifold $\tilde{L}[\kappa, \tau] \subset J^{1}\left(\mathbb{R}^{n+1}\right)$ parameterized by

$\tilde{\gamma}[\kappa, \tau](p, t)=\left(t, x(p), 2 \kappa(t-\tau) z(p), \kappa\left(1+(t-\tau)^{2}\right) \cdot y(p), \kappa\left(1+(t-\tau)^{2}\right) \cdot z(p)\right)$.

Note that the Reeb chords of $\tilde{L}[\kappa, \tau]$ correspond to the Reeb chords of $L$, lying in the slice $x_{0}=\tau$. In terms of fronts $\tilde{L}[\kappa, \tau]$ can be described as follows. Consider the front $F_{L} \subset J^{0}\left(\mathbb{R}^{n}\right)$ and the product $F_{L} \times \mathbb{R} \subset J^{0}\left(\mathbb{R}^{n+1}\right)$. The front $F_{\tilde{L}[\kappa, \tau]} \subset J^{0}\left(\mathbb{R}^{n+1}\right)$ is obtained by scaling the function coordinate $z$ of $F_{L} \times \mathbb{R}$ by a quadratic function on $\mathbb{R}$. The parameter $\tau$ determines where the scaling function has its minimum and the $t$-slices of the front grow as $t$ moves away from $t=\tau$. The parameter $\kappa$ determines the overall size of the scaling. Note, in particular, that the intersection of $\tilde{L}[\kappa, \tau]$ with the slice $t=\tau$ gives a copy of $L$.

Let $M$ be an $(n+1)$-manifold with boundary $\partial M=L_{-} \sqcup L_{+}$. Let $f: M \rightarrow J^{1}\left(\mathbb{R}^{n+1}\right)$ be a Legendrian embedding such that $\left|x_{0} \circ f\right| \leq 1$, such that $\left|x_{0} \circ f\right|<1$ in the interior of $M$, and such that $f$ agrees with $\gamma_{+}[\kappa,+1]: L_{+} \times(-\epsilon, 0]$ in some collar neighborhood $L_{+} \times(-\epsilon, 0]$ of $L_{+}$, and such that it agrees with $\gamma_{-}[\kappa,-1]: L_{-} \times[0, \epsilon)$ in some collar neighborhood $L_{-} \times[0, \epsilon)$ of $L_{+}$, where $\gamma_{ \pm}$are some Legendrian embeddings of $L_{ \pm}$. We say that $f(M)$ is a Legendrian embedding with standard ends. In the proof of Theorem 1.1, manifolds with boundary are used in order to concatenate isotopies and, in that way, express the contact homology of the trace of an isotopy subdivided into small pieces in a piece-by-piece manner.

If $f: M \rightarrow J^{1}\left(\mathbb{R}^{n+1}\right)$ is a Legendrian embedding with standard ends, then we can construct a Legendrian embedding $F$ of a non-compact manifold $\hat{M}$ obtained by adding $\gamma_{+}\left(L_{+} \times[0, \infty)\right)$ and $\gamma_{-}\left(L_{-} \times(-\infty, 0]\right)$ to $F$. Note that the Reeb chords of $F$ are exactly those of $f$. Moreover we have the following.

Lemma 3.4. Any holomorphic disk of finite area with boundary on $F(\hat{M})$ lies in the region $\left|x_{0}\right| \leq 1$ and the space of holomorphic disks is Gromov compact. Moreover any holomorphic disk with its positive puncture at $x_{0}= \pm 1$ lies entirely in this slice.

Proof. As in [7], projection to the $\left(x_{0}+i y_{0}\right)$-line in combination with the maximum principle establishes the first and third statements. The second then follows from the standard proof of Gromov compactness; see e.g. [5].

We have the corresponding statement for flow trees. 
Lemma 3.5. Any flow tree of $F(\hat{M})$ of finite symplectic area is contained in the region $\left|x_{0}\right| \leq 1$, and any flow tree with its positive puncture at $x_{0}= \pm 1$ stays entirely in this slice. Moreover, there is a 1-1 correspondence between, on the one hand, rigid holomorphic disks with boundary on $F(\hat{M})$ and, on the other, rigid flow trees determined by $F(\hat{M})$ and rigid flow trees of $L_{ \pm}$in slices.

Proof. The statements about flow trees follow from the fact that all gradient differences in the slices $x_{0}= \pm 1$ have trivial $x_{0}$-component. The second statement follows from a slight modification of the proof of [4, Theorem 1.1], which consists of two parts: convergence of holomorphic disks to flow trees and construction of holomorphic disks near rigid flow trees. The convergence part of the proof holds without change in this more general setting. The construction part can be subdivided into two parts: for trees not in the slices $x_{0}= \pm 1$ the proof from [4] applies. For trees in slices $x_{0}= \pm 1$ we apply the proof from [4] to $L_{ \pm}$thought of as lying in the slice.

Lemma 3.4 implies that the contact homology of $F(\hat{M})$ is well defined. (Here we restrict attention to Legendrian isotopies in the class of Legendrian submanifolds with standard ends). Lemma 3.5 implies that we can compute it using flow trees instead of holomorphic disks. We then define the contact homology of $f: M \rightarrow J^{1}\left(\mathbb{R}^{n+1}\right)$ to equal the contact homology of $F: \hat{M} \rightarrow$ $J^{1}\left(\mathbb{R}^{n+1}\right)$.

Remark 3.6. If $f_{j}: M_{j} \rightarrow J^{1}\left(\mathbb{R}^{n+1}\right)$ is a Legendrian submanifold with standard ends and if one of the ends of $\left(f_{0}, M_{0}\right)$ agrees with an end of $\left(f_{1}, M_{1}\right)$ then the two Legendrian embeddings can be joined to a Legendrian embedding $f_{01}$ of the manifold $M_{01}$ obtained by joining $M_{0}$ and $M_{1}$ along their common boundary. The contact homology differential of the join is determined in a straightforward way by the contact homology differentials of its pieces.

3.3. Generalized flow trees. In this section we introduce the notion of generalized flow trees for the product of a given Legendrian submanifold $L \subset J^{1}(\mathbb{R})$ and a manifold with boundary equipped with a Morse function. In the present paper we will apply this construction only in the case when the auxiliary manifold factor is an interval or a 2-disk.

Let $N$ be a manifold with boundary $\partial N$. We will use Morse functions $\beta: N \rightarrow \mathbb{R}$ of the following form. The restriction $\beta_{\partial}=\beta \mid \partial N$ is a Morse function on $\partial N$ and there is a collar neighborhood $\partial N \times[0, \epsilon)$ of $\partial N$ in $N$ where $\beta(x, t)=\beta_{\partial}(x)+k t^{2}$, where $(x, t) \in \partial N \times[0, \epsilon)$, and where $k>0$ is a constant. We call a Morse function of this type boundary adjusted.

Consider the Legendrian submanifold $L \times N \subset J^{1}(\mathbb{R} \times N)$. The Reeb chords of this Legendrian submanifold come in $N$-families, one for each Reeb chord of $L$. We denote the manifold of Reeb chords corresponding 
to the Reeb chord $c$ by $N_{c}$. Choose a boundary-adjusted Morse function $\beta_{c}: N_{c} \rightarrow \mathbb{R}$ for each Reeb chord manifold $N_{c}$. Endow $N$ with a Riemannian metric $g$ which has the following form in the collar neighborhood of the boundary:

$$
g(x, t)=g_{\partial}(x)+d t^{2},
$$

for $(x, t) \in \partial N \times[0, \epsilon)$, where $g_{\partial}$ is a Riemannian metric on $\partial N$.

A flow line $\gamma$ in a Reeb chord manifold $N_{c}$ is an oriented segment which can be parameterized in an orientation-preserving manner by a solution to the gradient equation

$$
\dot{q}=-\nabla \beta_{c}(q), \quad q \in N,
$$

where the gradient is defined using the Riemannian metric $g$ of (3.1). Note that a gradient line which starts in $\partial N_{c}$ stays in $\partial N_{c}$ and that a gradient line which starts in $N_{c}-\partial N_{c}$ can hit $\partial N_{c}$ only at a critical point in the boundary.

In order to define the notion of a generalized tree we first introduce some preliminary concepts. If $\Gamma$ is a flow tree of $L$ and if $q_{0} \in N$ then we let $\Gamma_{q_{0}}$ denote this flow tree considered as a flow tree of $L \times N$ and lying in the slice $\left\{(q, x) \in N \times \mathbb{R}: q=q_{0}\right\}$. We call $\Gamma_{q_{0}}$ a slice tree. A level is a finite collection $\Theta=\left\{\Gamma_{q_{1}}, \ldots, \Gamma_{q_{r}}\right\}$ of (unbroken) slice trees. A connector is a finite collection of flow lines $\theta=\left\{\gamma_{1}, \ldots, \gamma_{k}\right\}$ in Reeb chord manifolds, where we allow also flow lines of length 0 .

A generalized flow tree with $k$ levels is an ordered collection $\left(\Theta^{1}, \ldots, \Theta^{k}\right)$ of levels together with an ordered collection $\left(\theta^{0}, \ldots, \theta^{k}\right)$ of connectors which have the following properties.

- The connector $\theta^{0}$ consists of exactly one flow line in $N_{a}$ emanating at a critical point $p^{0} \in N_{a}$ of $\beta_{a}$ for some Reeb chord $a$ and ending at $q^{0} \in N_{a}$. If $k=0$ then $q^{0}$ is a critical point of $\beta_{\alpha}$ as well, and if $k>0$ then $q_{0}$ is not a critical point. Let $p^{1}=q^{0}$.

- The level $\Theta^{1}$ consists of exactly one slice tree $\Gamma_{p^{1}}$ with positive puncture at $a$.

- Let $j>0$, let $\left(\Gamma^{j, 1}\right)_{p_{1}^{j}}, \ldots,\left(\Gamma^{j, r}\right)_{p_{r}^{j}}$ denote the slice trees in the $j$ th level $\Theta^{j}$, and let $c_{1}^{j, s}, \ldots, c_{m_{s}}^{j, s}$ denote the Reeb chords at the negative punctures of $\Gamma^{j, s}, s=1, \ldots, r$. Then $\theta^{j}$ consists of flow lines emanating from those $p_{s}^{j} \in N_{c_{t}^{j, s}}, s=1, \ldots, r ; t=1, \ldots, m_{s}$, which are not critical points of $\beta_{c_{t}^{j, s}}$. Let $q_{1}^{j}, \ldots, q_{l}^{j}$ be the endpoints of the flow lines $\gamma_{1}^{j}, \ldots, \gamma_{l}^{j}$ in $\theta^{j}$ which are not critical points. Let $p_{s}^{j+1}=q_{s}^{j}$, $s=1, \ldots, l$.

- For $0<j \leq k, \Theta^{j+1}$ consists of slice trees $\left(\Gamma^{j+1,1}\right)_{p_{1}^{j+1}}, \ldots,\left(\Gamma^{j+1, r}\right)_{p_{l}^{j+1}}$ such that the positive puncture of $\Gamma^{j+1, s}$ is at the Reeb chord $a_{s}^{j+1}$ where $\gamma_{s}^{j} \subset N_{a_{s}^{j+1}}, s=1, \ldots, l$. 
- Let $q \in N_{c}$ be a point where some flow line $\gamma$ in a connector $\theta^{s}$, $s=0,1, \ldots, k$, ends. Then if there is no level tree $\Gamma_{q}$ in $\Theta^{s+1}$ with positive puncture matching the Reeb chord $c$, then $q$ is a critical point of $\beta_{c}$.

If $G$ is a generalized flow tree as just described, then we say that $G$ has positive puncture at the critical point of $a^{j} \in N_{a}$ of $\beta_{a}$ and negative punctures at all negative punctures of slice trees in $\Theta^{j}$ where no flow line in $\theta^{j}$ begins (these are critical points $b_{s}^{t} \in N_{b_{s}}$ ) and at the critical points $b_{s}^{t} \in N_{b_{s}}$ where some flow lines in some $\theta^{l}, 1 \leq l \leq k$, end. Any generalized flow tree has natural 1-jet and cotangent lifts. Adding suitably oriented capping paths at the punctures to the projection of the 1-jet lift of $G$ to $J^{1}(\mathbb{R})$, we get a homology class $A \in H_{1}(L ; \mathbb{Z})$. We write $\mathcal{G}_{A}\left(a^{j} ; b_{1}^{j_{1}}, \ldots, b_{m}^{j_{m}}\right)$ for the space of such generalized trees.

Example 3.7. Figure 2 shows a stabilized unknot $L$ with Reeb chords $a$ and $b$ and two of its rigid trees $\Gamma_{1}$ and $\Gamma_{2}$. The flow tree $\Gamma_{1}$ starts at $a$ as a flow line between sheets $A$ and $B$. This flow line splits over the leftmost cusp into two flow lines between $A$ and $C$ and between $C$ and $D$, respectively. The latter ends at $b$, the former splits over the middle cusp into two flow lines between $A$ and $D$ and $C$ and $D$, respectively. The latter ends at the rightmost cusp and the former ends at $b$. The flow tree $\Gamma_{2}$ starts at $b$ and splits immediately into two flow lines between $D$ and $C$ and $C$ and $B$, respectively. Both of these end in cusps.

Figure 3 shows a generalized 2-level tree of $L \times[0,1]$ : the first level is $\Gamma_{1}$ and the second is $\Gamma_{2}$.

As for flow trees we associate a planar domain and a map of its boundary into $T^{*} \mathbb{R}^{2}$ to any generalized flow tree. Using the map from the boundary of the planar domain we may endow the set of generalized flow trees with a topology. It is a straightforward consequence of the compactness properties

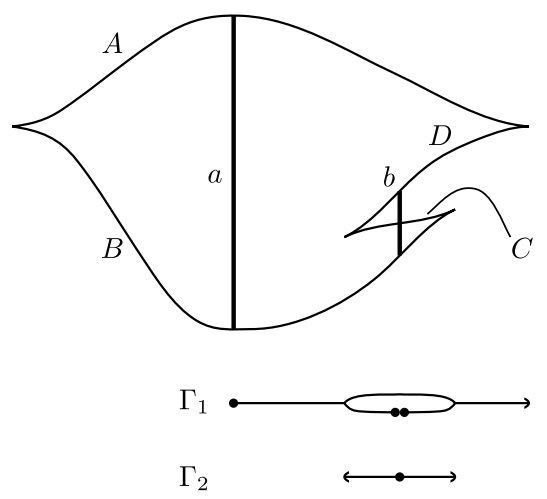

Figure 2. Two flow trees of a stabilized unknot. 


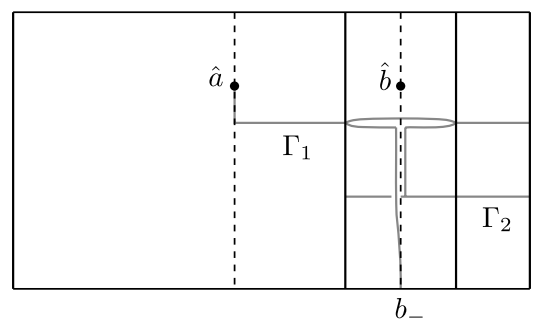

Figure 3. A 2-level flow tree of $L \times[0,1]$. Cusp edges are solid, Reeb chord manifolds are dashed.

of flow trees (see Lemma 3.2) that also the space of generalized flow trees has a natural compactification consisting of broken generalized flow trees. We denote the space of generalized flow trees by $\mathcal{G}$ and its compactification $\overline{\mathcal{G}}$.

3.4. Perturbed generalized flow trees. Let $L \subset J^{1}(\mathbb{R})$ be a Legendrian submanifold and let $N$ be a manifold with boundary. As in Section 3.3 we consider the Legendrian submanifold $L \times N \subset J^{1}(\mathbb{R} \times N)$ and we equip $N$ with a Riemannian metric and each Reeb chord manifold $N_{c}, c \in \mathcal{R}(L)$, with a Morse function $\beta_{c}$ satisfying conditions as stated there. Let $\overline{\mathcal{M}}$ denote the moduli space of flow trees on $L$ and fix a function $v: \overline{\mathcal{M}} \times N \rightarrow C^{k}\left(S^{1}, T N\right)$ with the following properties.

- $v(\Gamma, n) \in T_{n} N$ and $v(\Gamma, n)$ is tangent to $\partial N$ for $n \in \partial N$.

- By scaling lengths, we think of the source $S^{1}$ of $v(\Gamma, n)$ as the cotangent lift $\bar{\Gamma}$ of $\Gamma$ (with resolved self intersections). We require $v$ to be constant in neighborhoods of the punctures of $\bar{\Gamma}$ and equal to zero near the positive puncture. Consider a broken tree $\Gamma$ containing a broken tree $\Gamma^{\prime \prime}$. Let $\Gamma^{\prime}$ denote the broken tree obtained by removing $\Gamma^{\prime \prime}$ from $\Gamma$, and assume that $\Gamma^{\prime \prime}$ is attached to $\Gamma^{\prime}$ at a point $y$ in its cotangent lift. Then we require that for $x \in \Gamma^{\prime \prime}$ the following join equation holds

$$
v(\Gamma, n)(x)=v\left(\Gamma^{\prime}, n\right)(y)+v\left(\Gamma^{\prime \prime}, n\right)(x) .
$$

We call a function $v: \bar{M} \times N \rightarrow C^{k}\left(S^{1}, T N\right)$ with these properties a perturbation function.

The definition of a perturbed flow tree is analogous to that of a generalized flow tree. Fix a perturbation function $v$. If $\Gamma$ is a flow tree of $L$ and if $q \in N$, then recall that $\Gamma_{q}$ was used to denote the slice tree corresponding to $\Gamma$. Think of $\Gamma_{q}$ as a map $\Gamma_{q}=\left(\Gamma^{\mathbb{R}}, q\right): S^{1} \rightarrow \mathbb{R} \times N$, where $S^{1}$ is the cotangent lift of $\Gamma$ and the map $\left(\Gamma^{\mathbb{R}}(t), q\right)$ equals the natural map into $T^{*} \mathbb{R} \times T^{*} N$ followed by projection to $\mathbb{R} \times N$. We let $\tilde{\Gamma}_{q}$ denote the following map $S^{1} \rightarrow \mathbb{R} \times N$

$$
\tilde{\Gamma}_{q}(t)=\left(\Gamma^{\mathbb{R}}(t), \exp _{q}(v(\Gamma, q)(t))\right),
$$


where exp denotes the exponential map in a Riemannian metric of the form given in (3.1). We call $\tilde{\Gamma}_{q}$ a perturbed slice tree, and if $x$ is a negative puncture of $\Gamma$, then we write $e(q, x)=\exp _{q}(v(\Gamma, q)(x))$. A perturbed level is a finite collection $\tilde{\Theta}=\left\{\tilde{\Gamma}_{q_{1}}, \ldots, \tilde{\Gamma}_{q_{r}}\right\}$ of (unbroken) perturbed slice trees. A connector is a finite collection of flow lines $\theta=\left\{\gamma_{1}, \ldots, \gamma_{k}\right\}$ in Reeb chord manifolds, where we allow also flow lines of length 0 .

A perturbed flow tree with $k$ levels is an ordered collection $\left(\tilde{\Theta}^{1}, \ldots, \tilde{\Theta}^{k}\right)$ of levels together with an ordered collection $\left(\theta^{0}, \ldots, \theta^{k}\right)$ of connectors which have the following properties.

- The connector $\theta^{0}$ consists of exactly one flow line in $N_{a}$ emanating at a critical point $p^{0} \in N_{a}$ of $\beta_{a}$ for some $a \in \mathcal{R}(L)$ and ending at $q^{0} \in N_{a}$. If $k=0$ then $q^{0}$ is a critical point of $\beta_{\alpha}$ as well, if $k>0$ then $q_{0}$ is not a critical point. Let $p^{1}=q^{0}$.

- The level $\tilde{\Theta}^{1}$ consists of exactly one slice tree $\tilde{\Gamma}_{p^{1}}$ with positive puncture at $a$.

- Let $j>0$ and let $\left(\tilde{\Gamma}^{j, 1}\right)_{p_{1}^{j}}, \ldots,\left(\tilde{\Gamma}^{j, r}\right)_{p_{r}^{j}}$ denote the slice trees in the $j$ th level $\tilde{\Theta}^{j}$ and let $c_{1}^{j, s}, \ldots, c_{m_{s}}^{j, s}$ denote the Reeb chords at the negative punctures of $\Gamma^{j, s}, s=1, \ldots, r$. Then $\theta^{j}$ consists of flow lines emanating from those $e\left(p_{s}^{j}, x\right) \in N_{c_{t}^{j, s}}, s=1, \ldots, r ; t=1, \ldots, m_{s}$, where $x$ denotes the negative puncture corresponding to the Reeb chord $c_{t}^{j, s}$ which are not critical points of $\beta_{c_{t}^{j, s}}$. Let $q_{1}^{j}, \ldots, q_{l}^{j}$ be the endpoints of the flow lines $\gamma_{1}^{j}, \ldots, \gamma_{l}^{j}$ in $\theta^{j}$ which are not critical points. Let $p_{s}^{j+1}=q_{s}^{j}, s=1, \ldots, l$.

- For $0<j \leq k, \tilde{\Theta}^{j+1}$ consists of perturbed slice trees $\left(\tilde{\Gamma}^{j+1,1}\right)_{p_{1}^{j+1}}, \ldots$, $\left(\tilde{\Gamma}^{j+1, r}\right)_{p_{l}^{j+1}}$ such that the positive puncture of $\Gamma^{j+1, s}$ is at the Reeb chord $a_{s}^{j+1}$ where $\gamma_{s}^{j} \subset N_{a_{s}^{j+1}}, s=1, \ldots, l$.

- Let $q \in N_{c}$ be a point where some flow line $\gamma$ in a connector $\theta^{s}$, $s=0,1, \ldots, k$, ends. If there is no perturbed level tree $\tilde{\Gamma}_{q}$ in $\tilde{\Theta}^{s+1}$ with positive puncture matching the Reeb chord $c$, then $q$ is a critical point of $\beta_{c}$.

In analogy with generalized flow trees, we use the notion $\mathcal{G}_{\mathrm{p} ; A}\left(a^{j} ; b_{1}^{j_{1}}, \ldots\right.$, $\left.b_{m}^{j_{m}}\right)$ to denote the space of perturbed trees with specified punctures and boundary data as well as the notions $\mathcal{G}_{\mathrm{p}}$ for the space of all perturbed flow trees and $\overline{\mathcal{G}}_{\mathrm{p}}$ for its natural compactification consisting of several level trees.

Example 3.8. The top picture in Figure 4 shows a 1-level generalized flow tree of $L \times[0,1]$ where $L$ is the stabilized unknot as in Example 3.7. Applying the dimension formula for flow trees, we see that this tree has formal dimension -1 . The bottom picture shows a rigid 1-level tree arising from this configuration after perturbation by a generic perturbation function. 


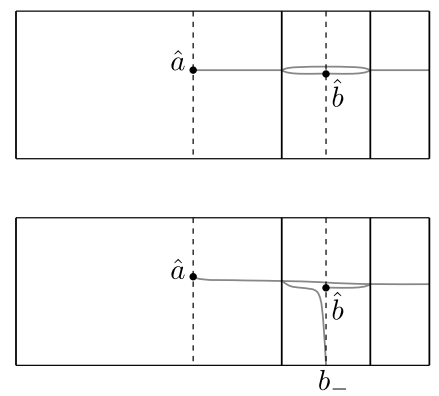

Figure 4. Generalized and perturbed flow trees.

Given a perturbation function and Morse functions on all Reeb chord manifolds, we associate a contact homology algebra with a differential to the Legendrian submanifold $L \times N$. The algebra $\mathcal{A}(L \times N)$ is the free $\mathbb{Z}_{2}\left[H_{1}(L ; \mathbb{Z})\right]$-algebra generated by critical points of the Morse functions $\beta_{c}$, for Reeb chords $c$, and the differential $\partial_{\mathrm{p}}: \mathcal{A} \rightarrow \mathcal{A}$ counts rigid perturbed flow trees. More precisely it satisfies the Leibniz rule and is defined as follows on generators:

$$
\partial_{\mathrm{p}} a=\sum_{\operatorname{dim}\left(\mathcal{G}_{\mathrm{p} ; A}\left(a ; b_{1}, \ldots, b_{k}\right)\right)=0}\left|\mathcal{G}_{\mathrm{p} ; A}\left(a, b_{1}, \ldots, b_{k}\right)\right| A b_{1} \cdots b_{k} .
$$

Lemma 3.9. For generic sufficiently small perturbations and Morse functions $\beta_{c}$, the moduli space of perturbed flow trees of formal dimension $\leq 1$ is transversely cut out. Consequently, $\partial_{\mathrm{p}}$ is a differential i.e., $\partial_{\mathrm{p}}^{2}=0$.

Proof. The transversality properties follows from standard applications of the finite-dimensional jet-transversality theorem. Furthermore, it is clear that, for small enough perturbation function, any sequence of perturbed rigid trees converges to a possibly broken perturbed rigid tree. The statement on the differential then follows from the usual gluing argument, which is technically very easy in this case since we need only glue Morse flow lines. (The perturbation function has to be sufficiently small so that for any $(\Gamma, n) \in \mathcal{M} \times N, v(\Gamma, n)$ is smaller than the injectivity radius of the Riemannian metric.)

We next concentrate on the case important for our applications. Let $N=I=[-1,1]$ and consider $L \times I$ equipped with two different perturbation functions $v^{0}$ and $v^{1}$ and with two different sets of Morse functions $\left\{\beta_{c}^{0}\right\}$ and $\left\{\beta_{c}^{1}\right\}$ where $c$ ranges over all Reeb chords. These data determine two different differentials $\partial_{0}$ and $\partial_{1}$ on $\mathcal{A}(L \times I)$. We show next that the resulting DGAs are tame isomorphic. We restrict the Morse functions so that they have minima at \pm 1 and exactly one interior maximum. We then write the 
algebra generators as $\{\hat{c}, c[-1], c[+1]\}$, where $\hat{c}$ denotes the maximum in $I_{c}$, $c[ \pm 1]$ the minima at $\pm 1 \in N_{c}$, and where $c$ ranges over all Reeb chords.

In order to see how the differentials $\partial_{0}$ and $\partial_{1}$ are related, we chose a generic path $\left(v^{s},\left\{\beta_{c}^{s}\right\}\right), 0 \leq s \leq 1$, of perturbations and Morse functions connecting the two sets of given data, where we restrict the Morse functions to be of the form described above. With such a path chosen, we get for each $s$ a moduli space $\mathcal{G}_{\mathrm{p}}^{s}$ of perturbed trees of $L \times I$.

Lemma 3.10. For a generic path, there exist no perturbed flow trees in $\mathcal{G}_{\mathrm{p}}^{s}$ of formal dimension $\leq-2$ and there exist finitely many instances where there are perturbed flow trees of formal dimension -1, and at such an instance, there is exactly one perturbed flow tree of dimension -1 which is transversely cut out in the sense of 1-parameter families.

Proof. This follows since the slice trees are transversely cut out and hence the only degeneration possible is when some incidence equation (involving the perturbation function and stable/unstable manifolds of the Morse functions) has non-transverse solutions.

Thus, for $s$ which is not a (-1)-tree instance, we get an induced differential $\partial_{s}$ on $\mathcal{A}(L \times I)$ by counting rigid perturbed trees.

Remark 3.11. We note that the appearance of a (-1)-tree as in Lemma 3.10 implies that many of the perturbed flow trees of formal dimension 0 are in fact appearing in higher dimensional families. These are obtained from rigid flow trees with some negative punctures at the positive puncture of the $(-1)$-disk by gluing. One way to relate the differentials is to study the details of how such families split as the deformation variable changes. Below we will however use a less explicit but technically simpler method.

To show that the DGAs $\left(\mathcal{A}(L \times I), \partial_{0}\right)$ and $\left(\mathcal{A}(L \times I), \partial_{1}\right)$ are tame isomorphic, we use a stabilization argument which is very close to the argument given in [7, Section 4.3]. Consider first the 1-parameter family of moduli spaces $\mathcal{G}_{\mathrm{p}}^{s}$ and let $\mathcal{G}_{\mathrm{p}}^{s}(0)$ denote the part of $\mathcal{G}_{\mathrm{p}}^{s}$ which consists of all perturbed trees of formal dimension 0 . If $s$ varies in an interval $\left[s_{0}, s_{1}\right]$ which does not contain any $(-1)$-tree instance, then the 1-manifold

$$
\cup_{s \in\left[s_{0}, s_{1}\right]} \mathcal{G}_{\mathrm{p}}^{s}(0)
$$

gives a cobordism between $\mathcal{G}_{\mathrm{p}}^{s_{0}}(0)$, and $\mathcal{G}_{\mathrm{p}}^{s_{1}}(0)$, and consequently $\partial_{s_{0}}=\partial_{s_{1}}$.

To show that $\partial_{0}$ and $\partial_{1}$ are tame isomorphic, it is thus sufficient to show that the DGAs on both sides of a (-1)-disk instance are tame isomorphic. We consider the product $L \times D$ where $D$ is a 2-disk. Think of the boundary of this disk as consisting of $I_{0}$ and $I_{1}$. Let $\beta_{c}^{0}$ and $\beta_{c}^{1}$ denote the Morse functions on the Reeb chord manifolds on the two sides of the $(-1)$-disk moment. Choose the Morse functions $\beta_{c}: D \rightarrow \mathbb{R}$ as extensions of these 


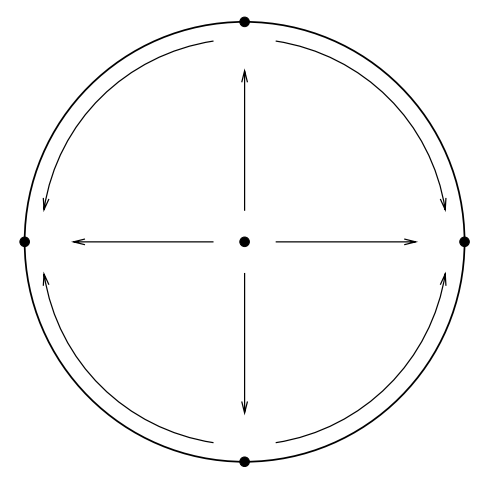

Figure 5. The Morse flow on the disk. Flow lines connecting $\tilde{c}$ to $\hat{c}[\sigma]$ and $c[\sigma]$, as well as $\hat{c}[\sigma]$ to $c[\sigma]$ are indicated.

functions with exactly one maximum and with Morse flows as shown in Figure 5.

Let furthermore the extension of the perturbation to the disk be that of the generic family, let $\tilde{\mathcal{A}}=\mathcal{A}(L \times D)$, and let $\Delta$ denote the corresponding differential. Write $\tilde{c}$ for the maximum Reeb chords, $\hat{c}[0]$ and $\hat{c}[1]$ for the saddle Reeb chords, and $c[0]$ and $c[1]$ for the minimum Reeb chords. Let $\Omega: \tilde{\mathcal{A}} \rightarrow \mathcal{A}$ be the algebra morphism which takes $\tilde{c}$ to $0, \hat{c}[\sigma]$ to $\hat{c}$, and $c[\sigma]$ to $c$.

Lemma 3.12. The differential $\Delta$ satisfies

$$
\begin{aligned}
\Delta c[\sigma] & =\left(\partial_{1} c\right)[\sigma]=\left(\partial_{0} c\right)[\sigma], \quad \sigma=0,1, \\
\Delta \hat{c}[\sigma] & =\left(\partial_{\sigma} \hat{c}\right)[\sigma], \quad \sigma=0,1, \\
\Delta \tilde{c} & =\hat{c}[0]+\hat{c}[1]+\epsilon+\mathbf{O}(1),
\end{aligned}
$$

where $\Omega \epsilon=m \alpha$, where $\alpha$ is the word of negative punctures of the $(-1)$-disk multiplied by the homology class of its boundary condition, where $m \in \mathbb{Z}_{2}$, and where $\mathbf{O}(1)$ denotes words which contain at least one $\tilde{c}$-variable.

Proof. The first two equations follow from the nature of the metric and the Morse function near the boundary. The second property follows from a limiting argument where we let the disk limit to the middle family containing the (-1)-disk. Any rigid disk must limit to a disk of formal dimension 0. All such disks which are not the $(-1)$-disk have at least one negative puncture at a $\tilde{c}$ variable.

Corollary 3.13. There exists a tame isomorphism between the algebras $\left(\mathcal{A}, \partial_{0}\right)$ and $\left(\mathcal{A}, \partial_{1}\right)$.

Proof. This follows from the fact that $\Delta^{2}=0$ in combination with (3.3). The details of the proof are found in [6, Lemma 4.21]. 


\section{Geometric perturbations of the trace of the constant isotopy}

In this section, we study the contact homology of Legendrian submanifolds which are traces of constant isotopies. We show that the contact homology differential can be expressed in terms of perturbed flow trees in this case.

4.1. Structure of the perturbation. Let $L \subset J^{1}(\mathbb{R})$ be a Legendrian submanifold with Reeb chords $c_{1}, \ldots, c_{m}$ and let $L \times I \subset J^{1}\left(\mathbb{R}^{2}\right)$ denote the trace of the constant isotopy starting and ending at $L$. For convenient notation we take $I=[-1,1]$ and we think of $L \times I \subset L \times \mathbb{R}$. Note that the Reeb chords of $L \times \mathbb{R}$ form 1-dimensional manifolds $\approx[-1,1]$, one for each Reeb chord of $L$. We will denote these manifolds $M_{c} \subset T^{*} \mathbb{R}^{2}$, where $c \in\left\{c_{1}, \ldots, c_{m}\right\}$ is the corresponding Reeb chord of $L$, and we will sometimes think of these manifolds as submanifolds of $J^{0}\left(\mathbb{R}^{2}\right)$. Furthermore, we will write $M_{c}^{+}$and $M_{c}^{-}$for the corresponding submanifolds of $L \times I$ of Reeb chord endpoints. In order to define the Legendrian contact homology of $L \times I$, we use any small perturbation which turns $L \times I$ into a Legendrian submanifold with standard ends and employ the definition from Section 3.2.

To get a more detailed understanding of the contact homology of $L \times I$, we will choose very specific perturbations. More precisely, we will design perturbations in four steps as follows:

(1) Make the ends of $L \times I$ standard.

(2) Make $\left|x_{0}\right|$ non-increasing along any flow line of a positive function difference of $L \times I$ and decreasing outside a neighborhood of the Reeb chord manifolds and outside a neighborhood of $\left\{x_{0}=0\right\}$.

(3) Make the Reeb chords isolated in such a way that there are three Reeb chords of $L \times I$ corresponding to each Reeb chord manifold, one at $x_{0}= \pm 1$ and one near $x_{0}=0$.

(4) Make $L \times I$ generic with respect to rigid flow trees.

The goal of this perturbation process is to obtain a description of the rigid trees needed to compute the contact homology of $L \times I$ in terms of perturbed flow trees of $L \times I$.

Steps (1)-(3) are straightforward to describe and are the subject of Section 4.2. Step (4) is more involved. It uses generalized flow trees, introduced in Section 3.3, and will be completed in Section 4.4.

4.2. Standard ends and isolated Reeb chords. Consider Step (1). Let $L \subset J^{1}(\mathbb{R})$ be given by

$$
a(q)=\left(x_{1}(q), y_{1}(q), z(q)\right), \quad q \in L .
$$

In coordinates $\left(x_{0}, y_{0}, x_{1}, y_{1}, z\right)$ on $J^{1}\left(\mathbb{R}^{2}\right)=T^{*} \mathbb{R}^{2} \times \mathbb{R}$, the unperturbed trace of the constant isotopy is then given by

$$
A(q, t)=\left(t, 0, x_{1}(q), y_{1}(q), z(q)\right), \quad(q, t) \in L \times I .
$$


Fix a smooth even function $\psi:[-1,1] \rightarrow \mathbb{R}$ with the following properties: $\psi(0)=1, \psi$ and all its derivatives vanish in $\left[0, \frac{1}{2}\right], \psi$ is decreasing on $\left[\frac{1}{2}, 1\right]$, and $\psi$ has a non-degenerate minimum at $1, \psi(1)=\frac{1}{2}$. Let $\dot{\psi}$ denote the derivative of $\psi$. For $\eta>0$, define the Legendrian embedding $A_{\eta}^{(\mathbf{1})}: L \times I \rightarrow$ $J^{1}\left(\mathbb{R}^{2}\right)$ by

$$
A_{\eta}^{(\mathbf{1})}(q, t)=\left(t, \eta \dot{\psi}(t) z(q), x_{1}(q), \eta \psi(t) y_{1}(q), \eta \psi(t) z(q)\right) .
$$

The Reeb chords of $A_{\eta}^{(\mathbf{1})}(L \times I)$ are of two types: isolated Reeb chords in $\left\{x_{0}= \pm 1\right\}$ at the locations of the Reeb chords of $L$ and non-isolated Reeb chords along the manifolds $\hat{M}_{c}=M_{c} \cap\left\{\left|x_{0}\right| \leq \frac{1}{2}\right\}$.

Consider Step (2). Fix a function $\phi:[-1,1] \rightarrow(-\infty, 0]$, with the following properties: $\phi$ is even, $\phi(0)=0, \phi$ is decreasing on $\left(0, \frac{1}{2}\right)$, and $\phi$ and all its derivatives vanish at $\left[\frac{1}{2}, 1\right]$. Consider a neighborhood $N\left(M_{c}^{ \pm}\right)$of a Reeb chord endpoint manifold $M_{c}^{ \pm}$which, under $\Pi: J^{1}\left(\mathbb{R}^{2}\right) \rightarrow \mathbb{R}^{2}$, maps to the product $\Pi\left(M_{c}\right) \times K$, where $K=\left\{x_{1}:\left|x_{1}-x_{1}(c)\right|<\delta\right\}$, where $x_{1}(c)$ is the $x_{1}$-coordinate of the Reeb chord $c$, for some small $\delta>0$. Consider a cut-off function $\hat{\xi}_{K}: K \rightarrow[0,1]$ which equals 0 on $\left|x_{1}-x_{1}(c)\right| \leq \frac{1}{2} \delta$ and equals 1 on $\frac{3}{4} \delta<\left|x_{1}-x_{1}(c)\right|<\delta$. Let $\hat{\xi}: M_{c} \times K \rightarrow[0,1]$ denote the function with $\hat{\xi} \mid\{p\} \times K=\hat{\xi}_{K}$ for every $p$. Consider the pull-back of $\hat{\xi}$ to $N\left(M_{c}\right)$ and note that it extends constantly to all of $L \times I$. Denote the extension $\xi$. Write

$$
A_{\eta}^{(\mathbf{1})}(q, t)=\left(t, y_{0}^{\eta}(q, t), x_{1}^{\eta}(q, t), y_{1}^{\eta}(q, t), z^{\eta}(q, t)\right),
$$

where $A_{\eta}^{(\mathbf{1})}(q, t)$ is as in (4.1). The function $\phi$ depends only on the $x_{0}$-coordinate and the function $\xi$ only on the $x_{1}$ coordinate. Denote their respective derivatives by $\dot{\phi}$ and $\dot{\xi}$. Define the Legendrian embedding $A_{\eta}^{(2)}: L \times I \rightarrow J^{1}\left(\mathbb{R}^{2}\right)$ by

$$
A_{\eta}^{(2)}(q, t)=\left(t,(1+\eta \xi \phi) y_{0}^{\eta}+\eta \dot{\xi} \dot{\phi}, x_{1}^{\eta},(1+\eta \xi \phi) y_{1}^{\eta}+\eta \dot{\xi} \phi,(1+\eta \xi \phi) z^{\eta}\right) .
$$

If $\gamma$ is a flow line of a Legendrian submanifold $H \subset J^{1}(M)$, write $\gamma^{+}$and $\gamma^{-}$ for the 1-jet lift of $\gamma$ with the larger and smaller $z$-coordinate, respectively.

Lemma 4.1. Let $\eta>0$ and let $p$ be a point on a flow line $\gamma$ of a positive function difference of $A_{\eta}^{(\mathbf{2})}(L \times I)$. Assume that $p$ does not lie in $\left\{x_{0}=0\right\}$ and that not both $\gamma^{+}$and $\gamma^{-}$lie in the region where $\xi=0$. Then $\left|x_{0}\right|$ is decreasing along $\gamma$ at $p$.

Proof. This follows from the choice of $\phi$ together with the fact that the $x_{0}$-component of $\nabla \xi$ is everywhere 0 .

Consider Step (3). In order to make Reeb chords of $L \times I$ isolated, we fix functions $\beta_{c}: M_{c} \rightarrow(-\infty, 0]$ for each Reeb chord $c$ of $L$. Consider $M_{c}=[-1,1]$ after projection to the $x_{0}$-line. Choose $\beta_{c}$ so that it has nondegenerate minima at \pm 1 , a non-degenerate maximum with value 0 close 
to $x_{1}=0$, and no other critical points. We assume moreover that the $x_{0}$-coordinates of the maxima of $\beta_{c}$ and $\beta_{c^{\prime}}$ are different if $c \neq c^{\prime}$. Furthermore, let $\xi$ be a function just like $\xi$ discussed above but replacing $\delta$ by $\frac{\delta}{100}$ and let $\alpha=1-\xi$. We pull back the functions $\beta_{c}$ to neighborhoods $N\left(M_{c}^{ \pm}\right)$ of the form describe above. Cutting these pull-backs off with $\alpha$, we find that the function $\alpha \sum_{c} \beta_{c}$ extends in an obvious way to all of $L \times I$. We denote the extension $\alpha \beta$. Similarly, the functions $\dot{\alpha} \beta=\dot{\alpha} \sum_{c} \beta_{c}$ and $\alpha \dot{\beta}=\alpha \sum_{c} \dot{\beta}_{c}$ may be considered as functions on all of $L \times I$. Write

$$
A_{\eta}^{(\mathbf{2})}(q, t)=\left(t, y_{0}^{\eta}(q, t), x_{1}^{\eta}(q, t), y_{1}^{\eta}(q, t), z^{\eta}(q, t)\right)
$$

where $A_{\eta}^{(\mathbf{2})}(q, t)$ is as in (4.2). Define $A_{\eta}^{(\mathbf{3})}: L \times I \rightarrow J^{1}\left(\mathbb{R}^{2}\right)$ by

$A_{\eta}^{(\mathbf{3})}(q, t)=\left(t,\left(1+\eta^{2} \alpha \beta\right) y_{0}^{\eta}+\eta^{2} \alpha \dot{\beta}, x_{1}^{\eta},\left(1+\eta^{2} \alpha \beta\right) y_{1}^{\eta}+\eta^{2} \dot{\alpha} \beta,\left(1+\eta^{2} \alpha \beta\right) z^{\eta}\right)$.

Then $A_{\eta}^{(\mathbf{3})}(q, t)$ has isolated Reeb chords as claimed in Step (3) above.

Example 4.2. The top picture in Figure 6 shows part of the front of a Legendrian knot $L$ which supports a rigid flow tree. The flow tree starts at $a$ as a flow line between sheets $A$ and $B$. This flow line splits into the Reeb chord $b$ and a flow line between sheets $A$ and $C$ which ends at $c$. The two bottom pictures show two perturbations of $L \times I$ which make its Reeb chords isolated as well as two rigid flow trees of the perturbed $L \times I$. Note that the flow trees depend in a crucial way on the choice of perturbation.
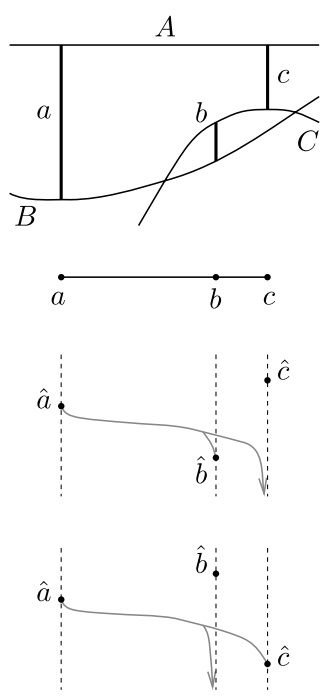

Figure 6. Flow trees and perturbations. 
4.3. Rough and fine scales - limits on the rough scale. As mentioned, we will consider the details of Step (4), where we introduce our final perturbation of $L \times I$ resulting in a Legendrian embedding $A_{\eta}^{(4)}: L \times I \rightarrow$ $J^{1}\left(\mathbb{R}^{2}\right)$, in Section 4.4. In this section, we will use rough properties of the perturbation of $A_{\eta}^{(4)}$ in order to derive rough results. More precisely, the property we use is the following.

- $A_{\eta}^{(4)}$ is a perturbation of $A_{\eta}^{(3)}$ of size $\mathbf{O}\left(\eta^{2}\right)$ which is supported in the region outside the fixed neighborhoods of the Reeb chord manifolds.

In order to describe rigid flow trees of $A_{\eta}^{(4)}(L \times I)$, we consider convergence of such trees on two scales. On the rougher scale, the important part of any flow trees concentrates in $x_{0}$-slices. This is fairly independent of the details of the perturbations in Step (4). However, around such a slice of concentration, we then re-scale the $x_{0}$-coordinate by $\eta^{-1}$ and study the corresponding microscopic limit as well. It is on this fine scale that the details of the perturbation in Step (4) manifest themselves.

Lemma 4.3. Let $\gamma_{\eta}, \eta \rightarrow 0$ be a sequence of flow lines of $A_{\eta}^{(4)}(L \times I)$. Then, as $\eta \rightarrow 0$, some subsequence of $\gamma_{\eta}$ converges to a broken flow line on $L \times I$ consisting of flow lines of $L$ in horizontal slices and vertical flow lines of $\beta_{c}$ along $M_{c}$.

Proof. Outside a neighborhood of $M_{c}$, the flow lines of $A_{\eta}^{(4)}(L \times I)$ obviously converge to horizontal curves which are parallel copies of flow lines of $L$. Inside a neighborhood of the manifolds $M_{c}$, the flow lines are as in a standard Morse-Bott situation, and any sequence of such flow lines converges to a combination of vertical flow lines of $\beta_{c}$ and horizontal flow lines.

Lemma 4.3 gives the local convergence on the rough scale. The following lemma describes the rough limits of flow trees of $A_{\eta}^{(\mathbf{4})}(L \times I)$ as $\eta \rightarrow 0$.

Lemma 4.4. Any sequence of flow trees, of $A_{\eta}^{(4)}(L \times I)$ of uniformly bounded formal dimension and which are not contained in $\left\{x_{0}= \pm 1\right\}$, has a subsequence that converges to a generalized flow tree on $L \times I$.

Proof. Note that any gradient difference along a cusp edge of $A_{\eta}^{(\mathbf{4})}(L \times I)$ is transverse to the cusp edge. In particular, the preliminary transversality condition is met, see [4, Subsection 3.1]. It is a consequence of $[\mathbf{4}$, Lemma 3.12 ] and the preliminary transversality condition that the number of edges and vertices of trees in such a sequence is uniformly bounded. Hence, by passing to a subsequence, we may assume that the topological type of the trees in the sequence remains constant. The lemma then follows by applying Lemma 4.3 to the edges of the trees in the sequence.

As a first step toward the description of rigid flow trees of $A_{\eta}^{(4)}(L \times I)$, we rule out some generalized flow trees as limits of sequences of rigid trees. 
Lemma 4.5. Consider a sequence $\Gamma_{\eta}$ of rigid flow trees (with fixed punctures) of $A_{\eta}^{(4)}(L \times I)$ which converges to a generalized flow tree $G$. Let $\Gamma_{t}^{r}, r=1, \ldots, m$, be the slice trees of $G$ and let $\hat{n}(G)$ denote the number of negative punctures of $G$ (or of $\Gamma_{\eta}$ ) which lie at $\hat{c} \in M_{c}$ for some $c$. Then

$$
\sum_{r=1}^{m}\left(\operatorname{dim}\left(\Gamma_{t}^{r}\right)+1\right)-\hat{n}(G)=0 .
$$

Proof. It follows from the dimension formula for flow trees that the formal dimension of a flow tree close to $G$ is given by the left-hand side of (4.3).

Note in particular that if the Legendrian knot $L$ is generic, then $\operatorname{dim}\left(\Gamma_{t}^{r}\right) \geq 0$ for all trees in (4.3).

4.4. Flow tree genericity. In this subsection, we present the details of the construction of $A_{\eta}^{(4)}: L \times I \rightarrow J^{1}\left(\mathbb{R}^{2}\right)$. The Legendrian submanifold $A_{\eta}^{(4)}(L \times I)$ will be generic with respect to rigid flow trees for all sufficiently small $\eta>0$ and its rigid flow trees will admit a description in terms of generalized flow trees with auxiliary data. In fact, we will first perturb $A_{\eta}^{(\mathbf{2})}$ outside a neighborhood of the Reeb chord manifolds in such a way that $A_{\eta}^{(4)}$ is obtained from the perturbed $A_{\eta}^{(\mathbf{2})}$ exactly as $A_{\eta}^{(\mathbf{3})}$ was obtained from the original $A_{\eta}^{(\mathbf{2})}$ in Section 4.2 .

To this end, we first describe the space of (partial) generalized trees near a slice tree. Let $a, b_{1}, \ldots, b_{k}$ be Reeb chords of $L$ and consider $[\tau-\delta, \tau+\delta] \subset$ $[-1,1]$, where $\tau=x_{0}(\hat{b})$ for $b=b_{j}$ some $j$. Then by our choice of functions $\beta_{c}: M_{c} \rightarrow \mathbb{R}$, the gradient of $\beta_{c}$ induces an orientation on $M_{c} \cap\left\{\left|x_{0}-\tau\right| \leq \delta\right\}$ for every $c \neq b$ provided $\delta>0$ is sufficiently small. Consider the compactified moduli space of trees

$$
\overline{\mathcal{M}}=\overline{\mathcal{M}}\left(a ; b_{1}, \ldots, b_{k}\right)
$$

of $L$ with positive puncture at $a$ and negative punctures at $b_{1}, \ldots, b_{k}$. The interior of this space is a manifold of dimension $d$. (Local coordinates can be obtained by the location of the branch points on the boundary of the corresponding holomorphic disks.) The boundary $\partial \overline{\mathcal{M}}$ of $\overline{\mathcal{M}}$ consists of products of lower-dimensional moduli spaces corresponding to broken disks. If $\mathcal{M}_{1} \times \mathcal{M}_{2} \times \ldots \times \mathcal{M}_{r}$ is such a product, then the positive puncture of $\mathcal{M}_{1}$ is $a$, the positive puncture of $\mathcal{M}_{j}$ agrees with a unique negative puncture of some $\mathcal{M}_{k}$, for $k<j$, the negative punctures which are not matched by some positive puncture are $b_{1}, \ldots, b_{k}$, and the dimensions $d_{j}$ of $\mathcal{M}_{j}$ satisfy

$$
\sum_{j=1}^{r} d_{j}+(r-1)=d .
$$

As with generalized flow trees, it is convenient to organize such a product of moduli spaces into levels, where $\mathcal{M}_{1}$ is the first level and all moduli spaces 
with positive puncture paired with a negative puncture of $\mathcal{M}_{1}$ constitute the second level, and, in general, all moduli spaces with positive puncture paired with a negative puncture of a moduli space of level $j$ constitute the $(j+1)$ th level. In this way, $\overline{\mathcal{M}}$ is a naturally stratified space (a manifold with boundary with corners endowed with a Kuranishi structure, see [12] for this notion). We will associate a $(d+1)$-dimensional stratified space of (partial) generalized flow trees to $\mathcal{M}$. We call this space $\mathcal{P}=\mathcal{P}\left(a ; b_{1}, \ldots, b_{k}\right)$. It is constructed from strata of $\overline{\mathcal{M}}$ exactly as the space of generalized flow trees except that gradient lines need not be complete, as follows. Consider first the top-dimensional stratum. We associate to this stratum the space $\mathcal{P}_{0}$ which consists of trees $\Gamma_{t}$ in $\mathcal{M}\left(a ; b_{1}, \ldots, b_{k}\right)$ viewed as a tree in a slice, $t \in[\tau-\delta$, $\tau+\delta]$. This is a $(d+1)$-dimensional space: $d$ dimensions for the tree and one dimension for the slice. Consider next a stratum $S$ with $k$ levels $L_{1}, \ldots, L_{k}$. We associate a $(d+1)$-dimensional space $\mathcal{P}_{S}$ of partial generalized trees to this stratum as follows. First consider the tree in $L_{1}$ as a slice tree $\Gamma_{t}$. For each negative puncture of $\Gamma_{t}$, which matches a positive puncture of some tree in $\mathcal{M}_{2}$, pick an oriented flow segment of the corresponding Reeb chord manifold and let the tree in the second level have its positive puncture where this flow line ends and continue in this way until $\mathcal{M}_{k}$ is reached. It follows from (4.4) that the dimension of $\mathcal{P}_{S}$ equals $(d+1)$. Moreover, the spaces constructed fit together in an obvious way to a $(d+1)$-dimensional space, which is our space $\mathcal{P}$. In a sense it is a resolution of $\overline{\mathcal{M}}$.

Our next objective is to define an evaluation map

$$
\mathrm{ev}^{F}: \mathcal{P}\left(a ; b_{1}, \ldots, b_{k}\right) \rightarrow M_{b_{1}} \times \ldots \times M_{b_{k}},
$$

where $F$ labels a specific perturbation of the Legendrian $L \times I$. This map is constructed inductively.

The starting point for the construction is the observation that it is easy to reconstruct actual flow trees of $A_{\eta}^{(\mathbf{2})}(L \times I)$, see Section 4.2, near flow trees in slices. More precisely, let $F: L \times I \rightarrow J^{1}\left(\mathbb{R}^{2}\right)$ be a deformation of $L \times I$. We are only interested in small perturbations, so let its size be $\mathbf{O}(\eta)$ as $\eta \rightarrow 0$. Assume further that the perturbation is supported in the complement of the Reeb chord manifolds $M_{c}$ (just like for $A_{\eta}^{(2)}$ in Section 4.2). Outside the neighborhood of the Reeb chord manifolds, the $x_{1}$-component of the gradient of any local function difference of $L \times I$ is bounded from below. It follows from this that the projection of any flow tree on $F(L \times I)$ for $\eta>0$ sufficiently small to a slice is a tree.

Conversely, given a tree $\Gamma^{\prime}$ in a slice, we can integrate it to a tree on $F(L \times I)$ for small $\eta$. The integration procedure is inductive and amounts to solving the gradient equation corresponding to the perturbation $F$ along the edges of the tree. For the $x_{1}$-component of the edge, this results in a re-parameterization and the $x_{0}$-component simply become non-constant along the edge. To extend the integration over the entire tree, we start 
integrating along the edge (edges) emanating from the positive puncture of the tree with the initial condition given by the $x_{0}$-coordinate of the slice of $\Gamma^{\prime}$ and follow the flow orientation. Inductively, the result of integrations along edges closer to the positive puncture in the tree gives initial conditions for the integrations along outgoing edges at any vertex in the tree. If $\Gamma^{\prime} \in$ $\mathcal{M}\left(a ; b_{1}, \ldots, b_{k}\right)$, if the punctures of $\Gamma$ are $p_{0}, p_{1}, \ldots, p_{k}$ with $p_{0}$ the positive puncture, and if $\Gamma^{\prime}$ is considered a tree in a slice with $x_{0}$-coordinate $\alpha$, then we write $\iota\left(p_{0}, p_{j}\right) \in M_{b_{j}}$ for the result of integration along $\Gamma^{\prime}$ at the negative puncture $p_{j}$, with initial condition given by the $x_{0}$-coordinate at the positive puncture $p_{0}$.

Let $\Gamma \in \mathcal{P}\left(a ; b_{1}, \ldots, b_{k}\right)$ be a partial generalized flow tree with levels $\left(\Gamma_{j}^{1}, \ldots, \Gamma_{j}^{s_{j}}\right), j=1, \ldots, r$. We define $\mathrm{ev}^{F}(\Gamma)$ as follows. Apply the integration procedure to the top level $\Gamma_{1}^{1}$, starting at the $x_{0}$-coordinate of its positive puncture in $M_{a}$. This gives points $\iota\left(p_{0}^{1}, p_{j}^{1}\right) \in M_{c_{j}}$ for all the negative punctures $p_{j}^{1}$ of $\Gamma_{1}^{1}$. If a negative puncture $p_{j}^{1}$ of $\Gamma_{1}^{1}$ is also a negative puncture of $\Gamma$, then define $\iota^{\prime}\left(p_{j}^{1}\right)=\iota\left(p_{j}^{1}\right) \in M_{c_{j}}$ to be the corresponding $M_{c_{j}}$-component of the evaluation map ev ${ }^{F}$. If a negative puncture $p_{k}^{1}$ of $\Gamma_{1}^{1}$ is not a negative puncture of $\Gamma$, then $\iota\left(p_{0}^{1}, p_{k}^{1}\right) \in M_{c_{k}}$ is the initial condition for the integration procedure of the second level tree attached at $p_{k}^{1}$. Continuing inductively in this way over all the levels, we get for each negative puncture $p_{j}$ of $\Gamma$, which is a negative puncture $q_{r}$ of some level tree in $\Gamma$ with positive puncture $q_{0}$, a point $\iota^{\prime}\left(p_{j}\right)=\iota\left(q_{0}, q_{s}\right) \in M_{b_{j}}$. Define

$$
\operatorname{ev}^{F}(\Gamma)=\left(\iota^{\prime}\left(p_{1}\right), \ldots, \iota^{\prime}\left(p_{k}\right)\right) \in M_{b_{1}} \times \ldots \times M_{b_{k}} .
$$

Let $b$ be a Reeb chord, let $\tau=x_{0}(\hat{b})$, and let $p_{0}, p_{1}, \ldots, p_{k}$ denote the punctures of $\Gamma \in \mathcal{P}\left(a ; b_{1}, \ldots, b_{k}\right)$. Let $J=\left\{p_{j_{1}}, \ldots, p_{j_{s}}\right\}$ be a subset of the punctures of $\Gamma$. Consider the subvariety $\Sigma_{J, \tau} \subset M_{b_{1}} \times \ldots \times M_{b_{k}}$,

$$
\Sigma_{J, \tau}=\left\{\left(t_{1}, \ldots, t_{k}\right): t_{j}=\tau, \text { for all } j \text { such that } p_{j} \in J\right\} .
$$

We show next that there are small perturbations $F$ of $A_{\eta}^{(\mathbf{2})}$ such that ev ${ }^{F}$ is transverse to $\Sigma_{J, \tau}$ for all small $\eta>0$. We will write $\operatorname{ev}_{J}^{F}$ for the evaluation map ev ${ }^{F}$ composed with the projection to $\Pi_{p_{j} \in J} M_{b_{j}}$ (i.e., to the product of the factors corresponding to punctures in $J$ ).

We start with the following preliminary lemma concerning a general fact about flow trees with only one positive puncture.

Lemma 4.6. Let $\Gamma$ be a flow tree of a Legendrian submanifold $L \subset J^{1}(M)$ with only one positive puncture and let $v$ be a vertex of $\Gamma$. Then any sheet of $L$ which contains some point $\tilde{v}$ in the 1-jet lift of $v$ contains the 1-jet lift of exactly two flow lines with endpoint at $\tilde{v}$.

Proof. We label a sheet by its corresponding local function. As mentioned above, each edge in a tree with one positive puncture is naturally oriented 
by using the negative gradient flow of the local function difference which is positive and with this convention exactly one edge at each vertex is oriented toward it (incoming flow line), all others away from it (outgoing flow lines).

Assume that some sheet, $f_{1}$ say, contains lifts of more than two flow lines. As the 1-jet lift is an oriented curve, there must exist at least two lifts which are oriented away from $\tilde{v}$. By the definition of a flow tree, there must exist matching edges oriented toward $\tilde{v}$ and at most one of these can be a lift of the incoming edge. The remaining one must come from an outgoing edge which thus is a flow between $f_{2}$ and $f_{1}$, where $f_{2}>f_{1}$. Continuing this argument with the matching lift of the 1 -jet lift segment in $f_{2}$ etc., we find that the tree has at least two incoming edges at $v$ since there are only finitely many sheets. This contradiction establishes the lemma.

Next we derive certain injectivity properties of flow trees of $A_{\eta}^{(\mathbf{2})}(L \times I)$.

Lemma 4.7. Assume that the set of points where two local gradient differences of $L$ agree is discrete (this is obviously an open condition on $L$ ). If $v$ is any vertex of a flow tree $\Gamma$ of $A_{\eta}^{(\mathbf{2})}(L \times I)$ with incoming edge $e^{i}$ and outgoing edges $e_{1}^{o}, \ldots, e_{m}^{o}$, then the self intersections of the 1-jet lift of $\Gamma$ near the 1-jet lift of $v$ form a discrete set.

Proof. Consider an arc in one of the sheets. It is a consequence of Lemma 4.6 that there is one incoming and one outgoing flow in this sheet. The genericity condition on $L$ implies that the flows of the corresponding gradients intersect only at a discrete set of points.

In order to establish the desired transversality, we will work with a re-scaled version of the map $\operatorname{ev}_{J}^{F}$,

$$
s_{\eta}\left[\operatorname{ev}_{J}^{F}\right]=\frac{1}{\eta}\left(\operatorname{ev}_{J}^{F}-(\tau, \ldots, \tau)\right): \mathcal{P} \rightarrow \mathbb{R}^{|J|},
$$

where $|J|$ denotes the cardinality of $J$. Here we will take $\eta \rightarrow 0$, and apply the Sard-Smale theorem. Let $\mathcal{F}$ be the space of perturbations of $L \times I$ as described. (To get a hold of this space, we can think of it as of the space of all functions on $L \times I$ supported outside a neighborhood of the $M_{c}$.) The evaluation map discussed above then gives a map

$$
\mathrm{ev}_{J}: \mathcal{F} \times \mathcal{P} \rightarrow M_{b} \times \cdots \times M_{b},
$$

with a re-scaling $s_{\eta}\left[\mathrm{ev}_{J}\right]$ analogous to that defined above.

Lemma 4.8. The map $s_{\eta}\left[\mathrm{ev}_{J}\right]$ is transverse to $0 \in \mathbb{R}^{|J|}$ in a neighborhood of $A_{\eta}^{(2)}$ for all sufficiently small $\eta>0$.

Proof. Since transversality is an open condition, we need only show that the differential is onto at any $(F, \Gamma) \in \mathcal{F} \times \mathcal{P}$ with $s_{\eta}\left[\operatorname{ev}_{J}^{F}\right](\Gamma)=0$. Fix such $(F, \Gamma)$. To prove surjectivity, we show that $\partial_{j}+\mathbf{O}(\eta)$ is in the image of the 
differential for each $j$, where $\partial_{j}$ is the tangent vector along the $j$ th factor in the product $\left(M_{b}\right)^{r}$.

Consider the $j$ th puncture $q_{j}$ mapping to $\tau \in M_{b}$. Assume for definiteness that $\tau<0$. Start at $q_{j}$ and follow $\Gamma$ in the direction opposite to the flow orientation. Then we meet a first edge $e$ which leaves the region $N\left(M_{b}\right)$ around $M_{b}$ where no perturbation is supported. Perturb this edge near the entrance point of $N\left(M_{b}\right)$ by deforming the Legendrian submanifold. We claim that if the region $N\left(M_{b}\right)$ is chosen small enough, then the only negative puncture in $\Gamma$, which appears after the edge $e$ in the flow orientation and which map to $\tau \in M_{b}$, is $q_{j}$. To see this, we use the fact that the distance between any two negative punctures in a tree is uniformly bounded from below, see Lemma 5.1, to conclude that to get to a negative puncture after $q_{j}$, we must follow some edge which leaves $N\left(M_{b}\right)$ and in such a region, integration along any edge induces a strict decrease in the $x_{0}$-coordinate. Thus, the $x_{0}$-coordinate is smaller than $\tau$ at any later puncture.

Consider next punctures $q_{r}$, which appear after $q_{j}$, map to $\tau \in M_{b}$ but lie on a lower level tree. For such punctures, we can remove the corresponding components of $\partial_{r}$ which the already introduced perturbation gives rise to by varying the length of the attaching flow line.

Finally, we must deal with punctures connected to $e$ only via vertices above $e$. (The perturbation could affect such a vertex.) Consider the vertices connected to $e$ by an edge attached as close to $e$ as possible. These vertices lie above the perturbation region in $e$. After Lemma 4.7, we know that $\Gamma$ has injective points in this region and it is therefore easy to compensate for the shifts induced by earlier perturbations. Using an obvious induction, we take care of all punctures in the tree above $q_{j}$. (Note that all perturbations needed for these additional punctures appear off the main stem connecting $q_{j}$ to the positive puncture and at $x_{0}$-levels above that of $e$.)

Corollary 4.9. For an open dense set of perturbations $F$ of $A_{\eta}^{(2)}$,

$$
\left(\mathrm{ev}_{J}^{F}\right)^{-1}((\tau, . \stackrel{r}{.}, \tau))
$$

where $\mathrm{ev}_{J}^{F}: \mathcal{P}\left(a ; b_{1}, \ldots, b_{k}\right) \rightarrow M_{b} \times \stackrel{r}{.} \times M_{b}, \operatorname{dim}\left(\mathcal{P}\left(a ; b_{1}, \ldots, b_{k}\right)\right)=d+1$ is a manifold of dimension $d+1-r$.

Proof. This is a standard application of the Sard-Smale theorem.

We next specialize to the situation of most importance to us. Consider a compactified moduli space $\overline{\mathcal{M}}\left(a ; b_{1}, \ldots, b_{k}\right)$ of flow trees on $L$ of dimension $d$ and with at least $m \geq d$ negative punctures mapping to $b$ (i.e., $b_{j}=b$ for at least $d$ indices $j)$. Consider the corresponding space $\mathcal{P}=\mathcal{P}\left(a ; b_{1}, \ldots, b_{k}\right)$ and let $J$ be a subset of the punctures of trees in $\mathcal{P}$ mapping to $M_{b}$ of cardinality $|J|=d$. Note that the map $s_{\eta}\left[\operatorname{ev}_{J}^{F}\right]: \mathcal{P} \rightarrow \mathbb{R}^{|J|}$ is continuous on the space $s_{\eta}[\mathcal{P}]$ obtained from $\mathcal{P}$ by re-scaling the lengths of all connector flow lines 
by $\eta^{-1}$. Moreover, it is clear that the closed subset $\left(s_{\eta}\left[\operatorname{ev}_{J}^{F}\right]\right)^{-1}(0) \subset s_{\eta}[\mathcal{P}]$, where $F$ is of size $\mathbf{O}(\eta)$, is bounded. Hence it is compact. Thus, Corollary 4.9 implies that, for generic $F,\left(\operatorname{ev}_{J}^{F}\right)^{-1}(\tau, \ldots, \tau)$ is empty if $|J|>d+1$ and is a compact 0-dimensional manifold if $|J|=d+1$. Furthermore, any point in such a 0 -dimensional manifold $\left(\operatorname{ev}_{J}^{F}\right)^{-1}(\tau, \ldots, \tau)$ corresponds to a point in the interior $\mathcal{M}=\mathcal{M}\left(a ; b_{1}, \ldots, b_{k}\right)$ of $\overline{\mathcal{M}}=\overline{\mathcal{M}}\left(a ; b_{1}, \ldots, b_{k}\right)$ for the following reason. Recall that the boundary of the $d$-dimensional $\overline{\mathcal{M}}$ consists of products of the form

$$
\mathcal{M}_{d_{1}} \times \cdots \times \mathcal{M}_{d_{r}}
$$

where $\mathcal{M}_{d_{j}}$ is a moduli space of dimension $d_{j} \geq 0$ and where

$$
d_{1}+\cdots+d_{r}+(r-1)=d .
$$

Thus if $\left(\mathrm{ev}_{J}^{F}\right)^{-1}(\tau, \ldots, \tau)$ intersects $\partial \overline{\mathcal{M}}$, then there are subsets $J_{l}, J=\cup_{l} J_{l}$ of negative punctures of trees in $\mathcal{M}_{d_{l}}$ for which $\operatorname{ev}_{J_{l}}^{F}(\tau, \ldots, \tau)$ is non-empty. The genericity condition then implies that $\left|J_{l}\right| \leq d_{l}+1$. Then $d-(r-1)=$ $\sum_{l} d_{l} \geq \sum_{l}\left|J_{l}\right|-1=|J|-1$ and $d+1>|J|$ since $r \geq 2$. This however contradicts $|J|=d+1$ and thus shows that all points lie in $\mathcal{M}$.

Let $p_{0}, p_{1}, \ldots, p_{k}$ denote the punctures of the generalized flow trees in $\mathcal{P}\left(a ; b_{1}, \ldots, b_{k}\right)$, where $\operatorname{dim}\left(\mathcal{M}\left(a ; b_{1}, \ldots, b_{k}\right)\right)=d$, let $F$ be a generic perturbation, let $J$ denote a subset of the punctures mapping to the Reeb chord $b$ with $|J|=d+1$, and let $x_{0}(\hat{b})=\tau$. We write $E_{J}^{F}\left(\overline{\mathcal{M}}\left(a ; b_{1}, \ldots, b_{k}\right)\right)=$ $\left(\operatorname{ev}_{J}^{F}\right)^{-1}(\tau, \ldots, \tau)$.

Remark 4.10. It is easy to see that if $\operatorname{dim}\left(\mathcal{M}\left(a ; b_{1}, \ldots, b_{k}\right)\right)=0$, then $E_{J}^{F}\left(\overline{\mathcal{M}}\left(a ; b_{1}, \ldots, b_{k}\right)\right)$ consists of exactly one point.

Since the moduli space of flow trees of $L$ is compact and since there are only finitely many Reeb chord manifolds, Lemma 4.8 implies that we can find a perturbation $F$ of $A_{\eta}^{(\mathbf{2})}$ which makes all evaluation maps $\mathrm{ev}^{F}: \overline{\mathcal{M}}\left(a ; b_{1}, \ldots, b_{k}\right)$ transverse to all varieties $\Sigma_{J, \tau}$, where $\tau=x_{0}(b)$ for some $b$ and where $J$ is any subset of the negative punctures which map to $b$, for all moduli spaces and all choices of collections of punctures.

We fix a choice of such an $F$ and perform also the perturbations of size $\mathbf{O}\left(\eta^{2}\right)$ along the Reeb chord manifolds which were used to obtain $A^{(\mathbf{3})}$ from $A^{(\mathbf{2})}$ in Section 4.2. Denote the resulting Legendrian embedding $A_{\eta}^{(\mathbf{4})}: L \times$ $I \rightarrow J^{1}\left(\mathbb{R}^{2}\right)$. We next give a description of the rigid flow trees of $A_{\eta}^{(4)}(L \times I)$ for small $\eta>0$.

Consider a generalized flow tree $G \in \mathcal{P}\left(a ; b_{1}, \ldots, b_{s}\right)$ on $L \times I$. A complete slice $S$ of $G$ is a collection of slice trees of $G$ of the following form.

- There is one slice tree $\Gamma^{0}$ in $S$ which lies on a level above (i.e., a level of lower numbering than) all other slice trees in $S$. 
- The positive puncture of $\Gamma^{0}$ is either the positive puncture of $G$ or connected to a negative puncture of some other slice tree in $G$ by a flow line of length $>0$.

- Every slice tree in $S$ of level $k$, which is lower than the level of the top slice tree $\Gamma^{0}$, is connected at its positive puncture via a flow line of length 0 to the negative puncture of some slice tree in $S$ of level $k-1$.

Thus, a complete slice $S$ is a (possibly broken) tree in a level lying in some compactified moduli space $\overline{\mathcal{M}}_{S}=\overline{\mathcal{M}}\left(e ; c_{1}, \ldots, c_{k}\right)$. If the complete slice $S$ lies in the $x_{0}$-slice of $\hat{c}$, where $c=c_{j}$ for one of the negative punctures $c_{j}$, then $E_{J}^{F}\left(\overline{\mathcal{M}}_{S}\right)$ may be non-empty for suitable $J$.

Consider a generalized flow tree $G \in \mathcal{P}\left(a ; b_{1}, \ldots, b_{s}\right)$ of $L \times I$ and let $S_{1}^{G}, \ldots, S_{k}^{G}$ be its complete slices. We say that $G$ is potentially rigid if for every $S_{k}^{G} \in \overline{\mathcal{M}}\left(e ; c_{1}, \ldots, c_{k}\right)$ in an $x_{0}(\hat{c})$-slice, $c=c_{j}$ for some $j$, the number of punctures mapping to $c$ (i.e., the number of indices $j$ such that $c=c_{j}$ ) is larger than $\operatorname{dim}\left(\mathcal{M}_{S_{j}^{G}}\right)$. In this case, we take $E^{F}\left(\overline{\mathcal{M}}_{S_{j}^{G}}\right)$ to be the union of all the manifolds $E_{J}^{F}\left(\overline{\mathcal{M}}_{S_{j}^{G}}\right)$ over all distinct choices of subsets $J$ of negative punctures with $|I|=d+1$.

Lemma 4.11. For all sufficiently small $\eta>0$, there is a $1-1$ correspondence between, on the one hand, rigid flow trees of $A_{\eta}^{(4)}(L \times I)$ and, on the other, the union over all potentially rigid generalized flow trees $G$ of the product sets

$$
E^{F}\left(\overline{\mathcal{M}}_{S_{1}^{G}}\right) \times E^{F}\left(\overline{\mathcal{M}}_{S_{2}^{G}}\right) \times \ldots \times E^{F}\left(\overline{\mathcal{M}}_{S_{k}^{G}}\right),
$$

where $S_{j}^{G}, j=1, \ldots, k$, are the complete slices of $G$.

Proof. Let $G$ be a potentially rigid generalized flow tree and let $F$ denote the perturbation of $A_{\eta}^{(2)}$ discussed above. A point in the product

$$
E^{F}\left(\overline{\mathcal{M}}_{S_{1}^{G}}\right) \times E^{F}\left(\overline{\mathcal{M}}_{S_{2}^{G}}\right) \times \ldots \times E^{F}\left(\overline{\mathcal{M}}_{S_{k}^{G}}\right)
$$

corresponds to $k$ complete slices of $G$, each of which gives a transverse solution to an equation $s_{\eta}\left[\mathrm{ev}_{J}^{F}\right]=0$ for some Reeb chord $b$ and some collection of punctures $J$. The Legendrian embedding $F$ is at $\mathbf{O}(\eta)$ distance from the inclusion of $L \times I$ and has Morse-Bott Reeb chords along $\hat{M}_{c}$ for all Reeb chords $c$ of $L$. To obtain $A_{\eta}^{(4)}$ from $F$, we make a perturbation of size $\mathbf{O}\left(\eta^{2}\right)$ in a small neighborhood of the manifolds $\hat{M}_{c}$.

The evaluation map is re-scaled by $\eta^{-1}$. After this re-scaling, the perturbation from $F$ to $A_{\eta}^{(4)}$ is a standard perturbation out of a Morse-Bott situation into a Morse situation. In particular, flow lines before the perturbation with evaluation map transversely equal to the location of the maximum, correspond in a 1-1 fashion to flow lines after the perturbation ending at the 
created maximum. We conclude that for $\eta>0$ sufficiently close to 0 , any point in the product gives rise to a collection of partial flow trees, with one negative puncture at a $\hat{c}$ for each negative puncture which maps to $x_{0}(\hat{c})$ by the evaluation map.

We show that these pieces can be glued in a unique way to a rigid flow tree of $A_{\eta}^{(4)}(L \times I)$. This is straightforward: by the definition of complete slice, all complete slices are connected to other complete slices by flow lines of length bounded from below. In order to glue the pieces, we thus need only make sure that the gluing problem for the flow lines of $\beta_{c}$ connecting the negative puncture at a complete slice of an above level to a complete slice in the level below has a unique solution. Consider first the case when the critical point is a maximum. Since the Morse-Bott perturbation is of size $\mathbf{O}\left(\eta^{2}\right)$, a change in the tree of the higher level near the negative puncture of size $\mathbf{O}\left(\eta^{2}\right)$ produces a finite change in the level of the outgoing flow line. Since the solution to $s_{\eta}\left[\mathrm{ev}_{c}^{F}\right]=0$ on the higher level is uniformly transverse, it persists under changes of size $\mathbf{O}\left(\eta^{2}\right)$. The case when the negative puncture has minimum character is similar: in order to connect the subtrees only, perturbations of the pieces of size $\mathbf{O}\left(\eta^{2}\right)$ are needed and the solutions to $s_{\eta}\left[\operatorname{ev}_{b}^{F}\right]=0$ persist under such perturbations. We conclude that near each point in the product (4.5), there is a unique rigid flow tree of $A_{\eta}^{(4)}(L \times I)$ for all $\eta>0$ sufficiently small.

In order to finish the proof, it remains only to check that any sequence of rigid trees of $A_{\eta}^{(4)}(L \times I)$ converges to a potentially rigid generalized tree and gives a solution to the evaluation condition of (4.5). The fact that it converges to a potentially rigid tree is an easy consequence of Lemma 4.4. Re-scaling by $\eta^{-1}$ takes us, as mentioned above, to a standard Morse-Bott situation near the Reeb chord manifolds, and it follows that any sequence of trees gives a solution.

We call the product of 0-dimensional manifolds as in (4.5) incidence spaces. Thus, the conclusion of Lemma 4.11 is that the contact homology differential of $A_{\eta}^{(4)}(L \times I)$, for $\eta>0$ sufficiently small, admits a description in terms of generalized flow trees and their corresponding incidence spaces. Although this is a rather nice geometric description, algebraically, it is in general messy. Moreover, even geometrically it is not very explicit: it is, in general, difficult to determine the incidence numbers since they require exact knowledge of moduli spaces of flow trees on $L$ of arbitrary dimension. (For a fixed knot $L$, this is less of a problem since any $L$ admits a presentation in which there are no disks with multiple negative punctures and for such knots the description of the algebra is straightforward.) However, we need to deal with 1-parameter families of knots and want the algebra as simple as possible, and as we shall see, there is an algebraically much preferable description of an algebra which is stable tame isomorphic to the one discussed above. 
This algebra arises from abstract perturbations and is related to the one above directly through its description in terms of generalized flow trees and incidence spaces. For this reason we note the following.

Remark 4.12. Fix a small $\eta>0$ for which Lemma 4.11 holds. Consider the Legendrian submanifold $F(L \times I)$, where $F$ is a small perturbation of

$A_{\eta}^{(2)}$. The integration along trees discussed above can be viewed as a map $\overline{\mathcal{M}} \times I \rightarrow C^{k}\left(S^{1}, T^{*} I\right)$, where $S^{1}$ is naturally identified with the cotangent lift of the tree. Lemma 4.11 then gives a description of the moduli space of rigid trees determined by $A_{\eta}^{(4)}(L \times I)$ in terms of perturbed trees and Morse functions $\beta_{c}: M_{c} \rightarrow \mathbb{R}$ on the Reeb chord manifolds, where the perturbation function is the $x_{0}$-component of the integration map discussed above.

\section{Abstract perturbations and contact homology computations}

In this section, we design a perturbation for the trace of the constant isotopy which leads to a simple differential.

5.1. A perturbation function. Let $\Gamma$ be a flow tree on $L \subset J^{1}(\mathbb{R})$. Note that the cotangent lift of $\Gamma$ is naturally parameterized by a map of a circle $S_{\Gamma}$ with metric induced by the parameterization and with ordered marked points $p_{0}, p_{1}, \ldots, p_{k}$ at the punctures of $\Gamma$. (Here $p_{0}$ corresponds to the positive puncture.) We will associate to $\Gamma$ a function $\phi_{\Gamma}: S_{\Gamma} \rightarrow \mathbb{R}$ such that $\phi_{\Gamma} \geq 0$ and such that

$$
\phi_{\Gamma}\left(p_{0}\right)=0=\phi_{\Gamma}\left(p_{k}\right)<\cdots<\phi_{\Gamma}\left(p_{1}\right) .
$$

Furthermore, the functions $\phi_{\Gamma}$ will vary continuously with $\Gamma$ in the moduli space of flow trees on $L$. In order to get a common source circle for all the maps $\phi_{\Gamma}$, we scale all cotangent lift circles $S_{\Gamma}$ so that they have length $2 \pi$ and view $\Gamma \mapsto \phi_{\Gamma}$ as a map $\overline{\mathcal{M}} \rightarrow C^{k}\left(S^{1}, \mathbb{R}\right)$, where $\overline{\mathcal{M}}$ is the compactified moduli space of trees of $L$ and where $k \geq 1$. Before discussing these functions, we prove a preliminary lemma.

Lemma 5.1. There exists a constant $C>0$ such that for any flow tree $\Gamma$ of $L$ with one positive puncture, the distance in $S_{\Gamma}$ between any two punctures is bounded below by $C$.

Proof. Let $c$ be a Reeb chord of $L$. The only Reeb chord in a neighborhood of $c$ is $c$ itself. For area reasons, if $c$ is the positive puncture of a tree, then it cannot also appear as a negative puncture in that tree. Thus it suffices to consider two neighboring negative punctures in a tree $\Gamma$ which both map to $c$. Consider the 1 -jet lift $\tilde{\Gamma}$ of $\Gamma$. Since the path of $\tilde{\Gamma}$ which connects the punctures is incoming at one of them and outgoing at the other, it connects the top endpoint of $c$ to the bottom endpoint of $c$ and thus its length is bounded from below. 
Assume that $L \subset J^{1}(\mathbb{R})$ is sufficiently generic so that the lengths of all its Reeb chords are pairwise distinct. Let the lengths be

$$
0<l_{1}<l_{2}<\cdots<l_{m} .
$$

It follows by the Stokes theorem that there exists $M$ (take $M>\frac{l_{m}}{l_{1}}$ ) such that no flow tree with one positive puncture has more than $M$ negative punctures, and that there exists $K$ (take $\left.K>\frac{l_{m}}{\min _{j}\left\{l_{j+1}-l_{j}, l_{1}\right\}}\right)$ such that no broken tree has more than $K$ levels.

Choose a function $h: \mathbb{R} \rightarrow \mathbb{R}, h>0$ such that for every $j=1, \ldots, r$

$$
\frac{1}{2 K M} h\left(l_{j+1}\right)>h\left(l_{j}\right) .
$$

We will define the map $\overline{\mathcal{M}} \rightarrow C^{k}\left(S^{1}, \mathbb{R}\right)$ in an inductive manner using the area filtration of $\overline{\mathcal{M}}$. In the first step, we define the map on the moduli space of flow trees of smallest area. In later steps, we define the map on the compactification of the moduli space of trees of higher areas assuming that the map is already defined for all moduli spaces of trees of smaller area. Since the compactification of a moduli space of trees of a given area consists of broken trees with pieces of smaller areas, the map has a natural definition on the boundary of the moduli space and we show how to extend it to the interior.

Let $\Gamma$ be a flow tree. We say that a smooth function $\phi_{\Gamma}: S_{\Gamma} \rightarrow \mathbb{R}$ is stretching if the following holds:

- If $\Gamma$ has one or zero negative punctures, then $\phi_{\Gamma}=0$.

- If $\Gamma$ has $r>1$ negative punctures and if $l$ is the length of its positive Reeb chord, then $\phi_{\Gamma}\left(p_{0}\right)=\phi_{\Gamma}\left(p_{r}\right)=0, \phi_{\Gamma}$ increases monotonically as we move along the circle in the negative direction from the point midway between $p_{r}$ and $p_{r-1}$ to the point $q$ midway between $p_{1}$ and $p_{0}$, $\phi_{\Gamma}$ decreases monotonically between $q$ and the point midway between $q$ and $p_{0}$ and then it is constant. Furthermore, $\phi_{\Gamma}\left(p_{j}\right)-\phi_{\Gamma}\left(p_{j+1}\right)=$ $\frac{1}{M} h(l)$ for $j=1, \ldots, r-1$.

Let

$$
\Gamma=\Gamma_{1}^{1} \cup\left(\Gamma_{2}^{1} \cup \ldots \cup \Gamma_{2}^{s_{2}}\right) \cup \ldots \cup\left(\Gamma_{l}^{1} \cup \ldots \cup \Gamma_{l}^{s_{l}}\right)
$$

be a broken tree where $\Gamma_{j}^{1}, \ldots, \Gamma_{j}^{t}$ are the trees of level $j$. Let $\phi_{\Gamma_{j}^{t}}: S_{\Gamma_{j}^{t}} \rightarrow \mathbb{R}$ be smooth functions which are constant in neighborhoods of punctures. Then these functions glue in an obvious way to a smooth function $\phi_{\Gamma}: S_{\Gamma} \rightarrow \mathbb{R}$ as follows. For points $x \in \Gamma_{1}^{1} \subset \Gamma$, let $\phi_{\Gamma}(x)=\phi_{\Gamma_{1}^{1}}(x)$. Assume inductively that $\phi_{\Gamma}$ has been defined for all points in trees of level $<j$. Let $x \in \Gamma_{j}^{t}$ and let $q_{j}^{t} \in \Gamma_{j-1}^{r}$ denote the negative puncture in the level $j-1$ tree $\Gamma_{j-1}^{r}$ where the positive puncture of $\Gamma_{j}^{t}$ is attached. Define $\phi_{\Gamma}(x)=\phi_{\Gamma}\left(p_{j}^{t}\right)+\phi_{\Gamma_{j}^{t}}(x)$. 
We define the common ancestor $A_{\Gamma}\left(q, q^{\prime}\right)$ of two negative punctures $q, q^{\prime}$ in the broken tree $\Gamma$ as an unbroken subtree of $\Gamma$, inductively, in the following way. If both $q$ and $q^{\prime}$ are negative punctures of the same unbroken tree $\Gamma_{j}^{t}$, then we take $A\left(q, q^{\prime}\right)=\Gamma_{j}^{t}$. If $q$ and $q^{\prime}$ lie on different levels $l(q)$ and $l\left(q^{\prime}\right)$ with $l(q)<l\left(q^{\prime}\right)$, say, then we take $A_{\Gamma}\left(q, q^{\prime}\right)=A_{\Gamma^{\prime}}\left(q, q^{\prime \prime}\right)$, where $q^{\prime \prime}$ is the negative puncture of the tree of level $l\left(q^{\prime}\right)-1$ where the positive puncture of the tree of $q^{\prime}$ is attached and where $\Gamma^{\prime}$ is the broken tree obtained from $\Gamma$ by cutting at $q^{\prime \prime}$. If $q$ and $q^{\prime}$ lie on the same level but not in the same tree, then we take $A_{\Gamma}\left(q, q^{\prime}\right)=A_{\Gamma^{\prime}}\left(p, p^{\prime}\right)$, where $p$ and $p^{\prime}$ are the negative punctures in the trees of level $l(q)-1=l\left(q^{\prime}\right)-1$ where the positive punctures of the trees of $q$ and $q^{\prime}$, respectively, are attached, and where $\Gamma^{\prime}$ is the broken tree obtained from $\Gamma$ by cutting at $p$ and at $p^{\prime}$.

Let $p_{0}, p_{1}, \ldots, p_{r}$ denote the punctures of a broken tree $\Gamma$ as in (5.1) .

Lemma 5.2. If $\phi_{\Gamma_{j}^{t}}$ are stretching for all $j$, then for any $t, 1 \leq t \leq r-1$,

$$
\phi_{\Gamma}\left(p_{t}\right)-\phi_{\Gamma}\left(p_{t+1}\right)>\left(1-\frac{L}{(K+1)}\right) \frac{1}{M} h\left(l\left(p_{t}, p_{t+1}\right)\right),
$$

where $L$ is the number of levels in $\Gamma$ and where $l\left(p_{t}, p_{t+1}\right)$ is the length of the Reeb chord of the positive puncture in $A_{\Gamma}\left(p_{t}, p_{t+1}\right)$.

Proof. We use induction. For trees of only one level this is immediate. Assume next that it holds for all broken trees of $L-1$ levels and consider attaching an $L$ th level to an $(L-1)$-level broken tree $\Gamma^{\prime}$ to form an $L$-level tree $\Gamma$. The only point that needs to be checked is that if $q$ is the first negative puncture in a tree $\Delta$ of level $L$ and if $p$ is the negative puncture in $\Gamma$ preceding it in $\Gamma$, then

$$
\phi_{\Gamma}(p)-\phi_{\Gamma}(q)>\left(1-\frac{L}{K+1}\right) \frac{1}{M} h(l(p, q)) .
$$

Note that either $p=p^{\prime}$, where $p^{\prime}$ lies in a tree of level at most $L-1$, or $p$ lies in a tree of level $L$ attached at a puncture $p^{\prime}$ of some tree of level $L-1$. In the latter case we have $\phi_{\Gamma}(p)=\phi_{\Gamma^{\prime}}\left(p^{\prime}\right)$. If $q^{\prime}$ denotes the negative puncture in the tree $\Gamma^{\prime}$ at which the tree $\Delta$ is attached, then the inductive assumption implies

$$
\phi_{\Gamma^{\prime}}\left(p^{\prime}\right)-\phi_{\Gamma^{\prime}}\left(q^{\prime}\right)<\left(1-\frac{L-1}{K+1}\right) \frac{1}{M} h\left(l\left(p^{\prime}, q^{\prime}\right)\right) .
$$


By the definition of common ancestor, $A\left(p^{\prime}, q^{\prime}\right)=A(p, q)$ and hence $h\left(l\left(p^{\prime}, q^{\prime}\right)\right)=h(l(p, q))$. Thus, since $\phi_{\Delta}$ is stretching, with $l=l(p, q)$,

$$
\begin{aligned}
\phi_{\Gamma}(p)-\phi_{\Gamma}(q) & =\phi_{\Gamma^{\prime}}\left(p^{\prime}\right)-\phi_{\Gamma^{\prime}}\left(q^{\prime}\right)-\phi_{\Delta}(q) \\
& >\left(1-\frac{L-1}{K+1}\right) \frac{1}{M} h(l)-M \frac{1}{2 K M} h(l) \\
& >\left(1-\frac{L-1}{K+1}-\frac{1}{K+1}\right) \frac{1}{M} h(l)=\left(1-\frac{L}{K+1}\right) \frac{1}{M} h(l) .
\end{aligned}
$$

Lemma 5.3. There exists a continuous function $\mathcal{M} \rightarrow C^{k}\left(S^{1}, \mathbb{R}\right), \Gamma \mapsto \phi_{\Gamma}$, such that the following holds. If $\Gamma$ is any (broken) tree in $\mathcal{M}$ with negative punctures $p_{1}, \ldots, p_{r}$, then

$$
\phi_{\Gamma}\left(p_{t}\right)-\phi_{\Gamma}\left(p_{t+1}\right)>\delta>0,
$$

and if $\Gamma$ is a broken tree, then $\phi_{\Gamma}$ satisfies the join equation (3.2).

Proof. Possible areas of trees of $L$ with one positive puncture constitutes a finite set of numbers

$$
0<\alpha_{1}<\alpha_{2}<\ldots<\alpha_{m}
$$

Let $\overline{\mathcal{M}}_{j}$ denote the compactification of the moduli space of trees of area $\leq \alpha_{j}$. Pick a stretching function $\Gamma \mapsto \phi_{\Gamma}$ for $\Gamma \in \mathcal{M}_{1}$. Assume inductively that we have defined a function $\overline{\mathcal{M}}_{j} \rightarrow C^{k}\left(S^{1}, \mathbb{R}\right)$ in such a way that there exists an $\epsilon>0$ with the following properties. In an $\epsilon$-neighborhood of each broken tree, the function satisfies the join equation. For $\Gamma$ outside a $2 \epsilon$-neighborhood of the boundary of $\overline{\mathcal{M}}_{j}$, the functions $\phi_{\Gamma}$ are stretching. In the region between these neighborhoods, the inequality,

$$
\phi_{\Gamma}\left(p_{t}\right)-\phi_{\Gamma}\left(p_{t+1}\right)>\frac{1}{2}\left(1-\frac{L}{(K+1)}\right) \frac{1}{M} h\left(l\left(p_{t}, p_{t+}\right)\right),
$$

where the notation is as in Lemma 5.2, holds. We want to extend the function to $\overline{\mathcal{M}}_{j+1}$. Since the boundary of $\overline{\mathcal{M}}_{j+1}$ consists of broken trees of area strictly smaller than $\alpha_{j+1}$, we define the map $\partial \overline{\mathcal{M}}_{j+1} \rightarrow C^{k}\left(S^{1}, \mathbb{R}\right)$ by imposing the join equation in a small neighborhood of the boundary of $\overline{\mathcal{M}}_{j+1}$. Using Lemma 5.2 , it is not hard to see that if $\epsilon>0$ is sufficiently small, then we can extend this family to all of $\overline{\mathcal{M}}_{j+1}$ respecting the above conditions on $\epsilon$-neighborhood as well as on the $2 \epsilon$-neighborhood and its complement. We obtain a function on $\overline{\mathcal{M}}$ with properties as desired.

We view a function $\overline{\mathcal{M}} \rightarrow C^{k}\left(S^{1}, \mathbb{R}\right)$ with properties as in Lemma 5.3 as a function into $C^{k}\left(S^{1}, T^{*} I\right)$ by identifying $\mathbb{R}$ with the fiber in $T^{*} I$. Fix such a function and use it as a perturbation function for flow trees, see Section 3.4. Let $\Delta_{\mathrm{a}}$ denote the corresponding differential on $\mathcal{A}(L \times I)$. 
Remark 5.4. By scaling, the total variation of any function $\phi_{\Gamma}, \Gamma \in \overline{\mathcal{M}}$ can be assumed arbitrarily small.

Lemma 5.5. Any rigid perturbed flow tree has only one level. The flow tree corresponding to the perturbed slice tree in that level is itself rigid and exactly one of its negative punctures lies at the 0-level, or the tree is entirely contained in $\left\{x_{0}= \pm 1\right\}$.

Proof. This follows from the choice of abstract perturbation. Since it orders the negative punctures, at most one at a time can lie at the 0-level. The lemma is then an easy consequence of the dimension formula.

Let $\partial: \mathcal{A}(L) \rightarrow \mathcal{A}(L)$ denote the contact homology differential and let the notation for generators be $\hat{c}$ for the maximum in $I_{c}$ and $c[ \pm 1]$ for the minima at $\pm 1 \in I_{c}$, for any Reeb chord $c$ of $L$.

Corollary 5.6. The differential $\Delta_{\mathrm{a}}: \mathcal{A}(L \times I) \rightarrow \mathcal{A}(L \times I)$ satisfies the following

$$
\begin{aligned}
\Delta_{\mathrm{a}} c[ \pm 1] & =\partial c[ \pm 1], \\
\Delta_{\mathrm{a}} \hat{c} & =c[+1]+c[-1]+\Gamma(\partial c),
\end{aligned}
$$

where $\Gamma(1)=0$ and

$$
\begin{aligned}
\Gamma\left(a_{1} a_{2} \cdots a_{m}\right)= & \hat{a}_{1} a_{2}[-1] \cdots a_{m}[-1] \\
& +a_{1}[+1] \hat{a}_{2} a_{3}[-1] \cdots a_{m}[-1] \\
& \vdots \\
& +a_{1}[+1] \cdots a_{m-1}[+1] \hat{a}_{m} .
\end{aligned}
$$

Proof. It is easy to construct the rigid perturbed trees mentioned in Lemma 5.5, which give the second term in (5.3), as well as the flow lines of $\beta_{c}$ which give the first term in (5.3). Lemma 5.5 shows that there are no other rigid perturbed trees.

Let $\Delta: \mathcal{A}(L \times I) \rightarrow \mathcal{A}(L \times I)$ denote the differential on the contact homology algebra of the trace of the constant isotopy which arises from a geometric deformation.

Corollary 5.7. There exists a tame isomorphism between the algebras $(\mathcal{A}(L \times I), \Delta)$ and $\left(\mathcal{A}(L \times I), \Delta_{\mathrm{a}}\right)$.

Proof. Remark 4.12 shows that $\Delta$ can be expressed as a differential $\Delta_{\mathrm{g}}$ induced by a perturbation function and by collections of Morse functions on the Reeb chord manifolds. Corollary 3.13 then shows that $\left(\mathcal{A}(L \times I), \Delta_{\mathrm{a}}\right)$ and $\left(\mathcal{A}(L \times I), \Delta_{\mathrm{g}}\right)$ are tame isomorphic. 


\section{Decomposing isotopies and differentials}

In this section, we first discuss how to subdivide a Legendrian isotopy into pieces in such a way that each piece corresponds either to a sufficiently good approximation of a constant isotopy or to a move isotopy which is constant except in a small region where it has one of the several standard forms. The computations of Section 5 allow us to describe the DGA of the trace of the almost constant isotopy, see Section 6.1. Slight extensions of these computations allow us to describe the DGAs of traces of move isotopies as well, see Sections 6.2 and 6.3.

6.1. The trace of an almost constant isotopy. Let $L \subset J^{1}(\mathbb{R})$ be a generic Legendrian submanifold. Let $L_{t},-1 \leq t \leq 1$, be an isotopy of $L=L_{0}$ and let $\Phi(L \times I)$ be the trace of $L_{t}$ with standard ends. Let $\Psi: J^{1}(\mathbb{R} \times$ $I) \rightarrow J^{1}(\mathbb{R} \times I)$ be the perturbation which makes the trace $L \times I$ of the constant isotopy generic with respect to flow trees.

Lemma 6.1. There exists $\epsilon=\epsilon(L)>0$ such that if the isotopy $L_{t}$ is contained in a the $C^{2} \epsilon$-neighborhood of the constant isotopy, then the contact homology algebra of $\Psi(\Phi(L \times I))$ and that of $\Psi(L \times I)$ are canonically isomorphic.

Proof. Assume not. Then there exists a sequence of isotopy traces $\Phi_{j}: L \times$ $I \rightarrow J^{1}(\mathbb{R} \times I), j=1,2, \ldots$, such that the space of rigid flow trees of $\Psi\left(\Phi_{j}(L \times I)\right)$ and that of $\Psi(L \times I)$ are non-isomorphic and such that $\Phi_{j} \rightarrow$ id as $j \rightarrow \infty$. By the compactness properties of the space of flow trees, this contradicts the flow trees of $\Psi(L \times I)$ being transversely cut out .

Let $L_{t}, 0 \leq t \leq 1$, be a Legendrian isotopy. It is well known that we may deform this isotopy into an isotopy with the property that $L_{t}$ is a generic Legendrian submanifold for $t \neq t_{j}$ where $0<t_{1}<\cdots<t_{m}<1$ is a finite collection of instances and such that around every $t_{j}$ the isotopy is constant outside a small disk and inside this disk the Legendrian undergoes one of the following standard moves.

(F1) A first Redemeister front move.

(F2) A second Redemeister front move.

(F3) A third Redemeister front move.

(L1) A Lagrangian triple point move.

(L2) A pair of Reeb chords disappears.

(L3) A pair of Reeb chords appears.

We call such an isotopy an isotopy with standard moves.

Given an isotopy with standard moves, we may subdivide the intervals between the standard moves in sufficiently short isotopies through generic Legendrian submanifolds. This gives a representative of the trace of the isotopy which is a join of approximately constant isotopies and isotopies 
with standard moves. Let $L$ be the middle stage of an almost constant isotopy and let $\{\hat{c}, c[-1], c[+1]\}$ denote the Reeb chords of the trace of the almost constant isotopy, where $c$ ranges over the Reeb chords of $L$, where $\hat{c}$ corresponds to the maximum in $I_{c}$ and $c[ \pm 1]$ to the minima at the endpoints of $I_{c}$. If $w \in \mathcal{A}(L)$ is an element, then write $w[ \pm 1]$ for the corresponding element in $\mathcal{A}(L \times I)$ where every generator $c$ has been replaced by $c[ \pm 1]$.

Corollary 6.2. The DGA differential $\Delta$ of the trace of an almost constant isotopy is the following

$$
\begin{aligned}
\Delta c[ \pm 1] & =(\partial c)[ \pm 1], \\
\Delta \hat{c} & =c[-1]+(\operatorname{id} c)[+1]+\Gamma_{\mathrm{id}}(\partial c),
\end{aligned}
$$

for every Reeb chord c.

Proof. This follows from Lemma 3.12 in combination with Lemma 6.1.

Concatenation of the almost constant isotopies of an isotopy without moves then gives an expression for the differential of the trace of the total isotopy.

6.2. Front moves. In this subsection, we study moves (F1)-(F3). In fact, none of these moves changes the differential from that of the trace of the constant isotopy.

Lemma 6.3. The DGA of the trace of an (F1)-move is identical to the $D G A$ of the trace of an almost constant isotopy, see (6.1).

Proof. We parameterize the trace by keeping the isotopy constant on $x_{0} \in$ $\left[-1, \frac{1}{2}\right]$, then performing the move, and keeping it again constant close to $x_{0}=1$. It is straightforward to check that there is a natural 1-1 correspondence between flow trees of the trace of the constant isotopy and flow trees of the (F1)-isotopy. The only difference between the two kinds of trees appears as they pass the bifurcation region where trees in one of the situations are obtained from trees in the other situation by adding a $Y_{1}$-vertex and an end, see Figure 7.

Lemma 6.4. The DGA of the trace of an (F2)-move is identical to the $D G A$ of the trace of an almost constant isotopy, see (6.1).
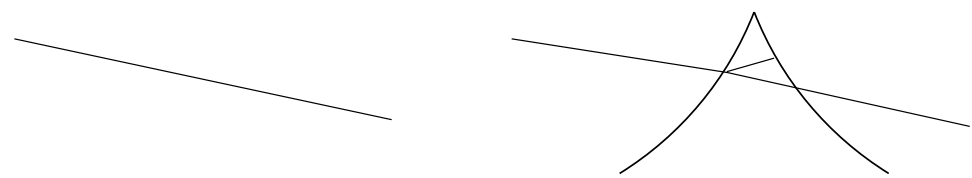

Figure 7. Flow line of the trace of the constant isotopy (left) and corresponding flow tree of the (F1)-isotopy (right). 
Proof. As in the proof of Lemma 6.3, it is straightforward to find a 1-1 correspondence between rigid flow trees of the trace of the constant isotopy and the trace of the isotopy of the move. To see this, use the cotangent lift of the trees and the fact that the Lagrangian projection of the trace of an (F2)-isotopy is qualitatively indistinguishable from that of the constant isotopy.

Lemma 6.5. The DGA of the trace of an (F3)-move is identical to the $D G A$ of the trace of an almost constant isotopy, see (6.1).

Proof. The same argument as in the proof of Lemma 6.4 applies.

6.3. Lagrangian moves. In this subsection, we study the moves (L1)(L3). To this end we will append the move isotopy to one end of a constant isotopy. As in Section 3.4, our computation uses abstract perturbations. That is, we use perturbed flow trees in our computations. The argument which shows that this computation gives a DGA which is tame isomorphic to the DGA which arises from a geometric perturbation is almost identical to the proof of Lemma 3.12 and Corollary 3.13 and will not be repeated.

Let $L_{-1}$ and $L_{+1}$ be Legendrian submanifolds of $J^{1}(\mathbb{R})$ such that $L_{+1}$ is obtained from $L_{-1}$ via an $(\mathbf{L j})$-move. Then we write $\phi_{L j}: \mathcal{A}\left(L_{-1}\right) \rightarrow \mathcal{A}\left(L_{+1}\right)$ for the induced homomorphism, $\mathbf{j}=\mathbf{1 , 2 , 3}$, see $[\mathbf{1 5}]$. Furthermore we write $\partial_{ \pm}$for the differential on $\mathcal{A}\left(L_{ \pm 1}\right)$ and if $\phi: \mathcal{A}\left(L_{-1}\right) \rightarrow \mathcal{A}\left(L_{+1}\right)$ is a homomorphism, we use the notion $\Gamma_{\phi}: \mathcal{A}\left(L_{-1}\right) \rightarrow \mathcal{A}(L \times I)$ as in Theorem 1.1.

We first consider the simpler cases of (L1) and (L2) where the abstract perturbations and the move region can be taken disjoint. We let the move happen inside a box of the form $\frac{1}{2} \leq x_{0} \leq \frac{1}{2}+\delta,\left|x_{1}-a\right| \leq \epsilon$. Inside this box, we can draw the flow explicitly and from that information compute the differential.

Lemma 6.6. The differential $\Delta$ of the trace of a move isotopy of type (L1) satisfies

$$
\Delta \hat{c}=c[-1]+\phi_{\mathrm{L} 1}(c)[+1]+\Gamma_{\phi_{\mathrm{L} 1}}\left(\partial_{-} c\right) .
$$

Proof. We chose abstract perturbations of the same form as in Section 3.4 and we use a cut-off function supported above the move region. As there, we find that as $\eta \rightarrow 0$ outside the move region, any sequence of trees converges to a generalized tree. In particular, any rigid flow tree has all its limit slice trees near $x_{0}=0$. The difference arises as the descending flow lines near the Reeb chord manifolds enter the box of the move. There are two different (L1)-moves, see Figures 8 and 9. The corresponding flow pictures are shown in Figures 10 and 11. It is clear from these pictures that there is no effect on the differential in the first case and that in the second case we get exactly $c[-1]+\phi(c)[+1]+\Gamma_{\phi}(\partial c)$ in the right hand side of $(6.2)$, where $\phi(a)=a+b c$ and $\phi$ is the identity on other generators. 

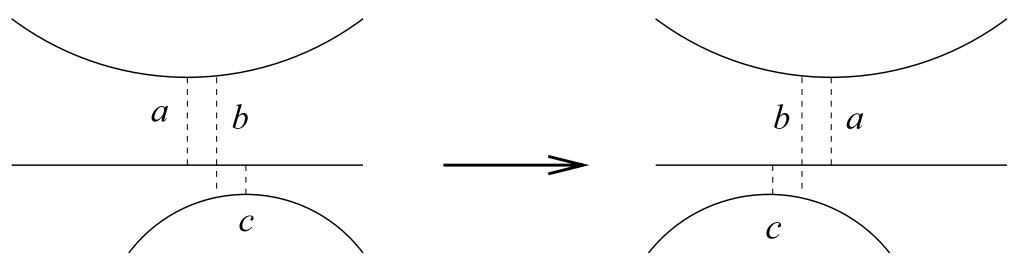

Figure 8. The first (L1)-isotopy.
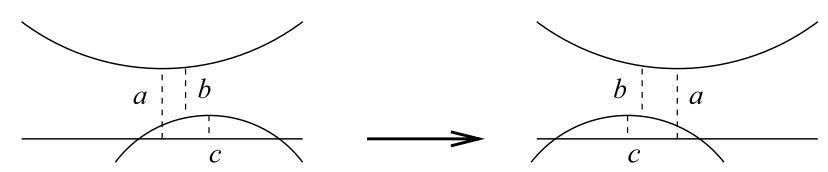

Figure 9. The second (L1)-isotopy.

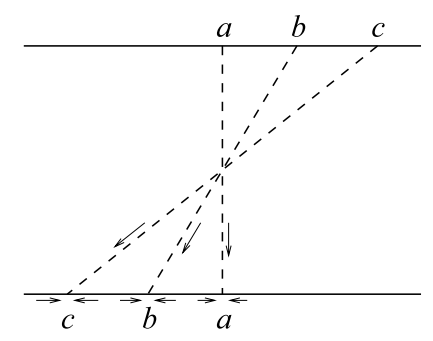

Figure 10. The trace of the first (L1)-isotopy. Every flow line belonging to a rigid tree passes right through the isotopy box.

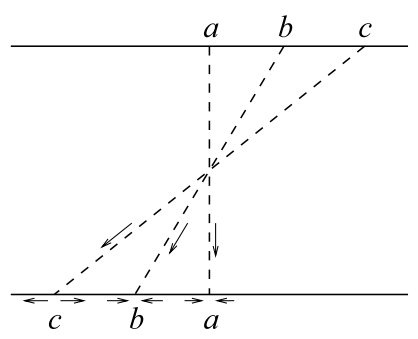

Figure 11. The trace of the second (L1)-isotopy. Any flow line on its way toward $a$ can either split over a $Y_{0}$-vertex into a rigid configuration of a flow line toward $b$ and a flow line toward $c$ or pass right through the isotopy box. 
Lemma 6.7. The differential $\Delta$ of the trace of a move isotopy of type (L2) satisfies

$$
\Delta \hat{c}=c[-1]+\phi(c)[+1]+\Gamma_{\phi_{\mathrm{L} 2}}\left(\partial_{-} c\right)
$$

Proof. The proof is very similar to the proof of Lemma 6.6. We use abstract perturbations supported outside the move box. The move is depicted in Figure 12 and the corresponding flow box in Figure 13. It follows that

$$
\Delta \hat{c}=c[-1]+\phi_{\mathrm{L} 2}(c)[+1]+\Gamma_{\phi_{\mathrm{L} 2}}\left(\partial_{-} c\right),
$$

where $\phi$ takes $a$ to $0, b$ to $\partial a+b=v$, and is the identity on all other generators.

Lemma 6.8. The differential $\Delta$ of the trace of a move isotopy of type (L3) satisfies

$$
\Delta \hat{c}=c[-1]+\phi_{\mathrm{L} 3}(c)[+1]+\Gamma_{\phi_{\mathrm{L} 3}}(\partial c) .
$$

Proof. To prove this result, we use an abstract perturbation of the kind discussed in Section 5, which orders the punctures according to their natural boundary ordering. This time we let the perturbation of the horizontal trees be cut-off only after the flow box of the move. This flow box is depicted in Figure 14. Our choice of perturbation guarantees that the flow lines of

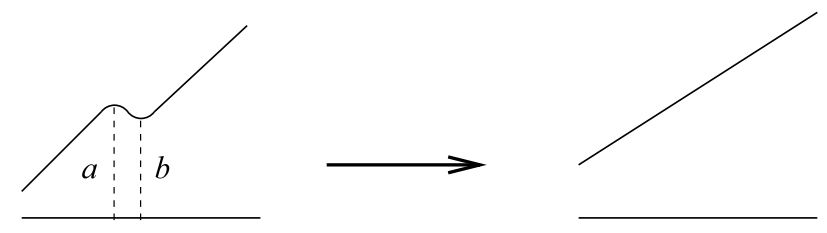

Figure 12. An (L2)-isotopy.

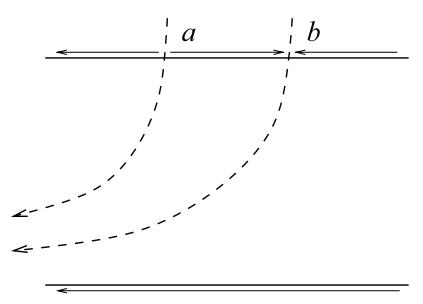

Figure 13. The trace of the (L2)-isotopy. Any flow line on its way toward $a$ goes on toward a word contributing to $v$. The corresponding tree lives in a 1-parameter family since nearby flow lines (which would formerly be attracted by $b$ ) also go on toward the same word in $v$. A flow line of a rigid tree on its way toward $b$ is determined uniquely already in the region above. It goes on to a word contributing to $v$. 


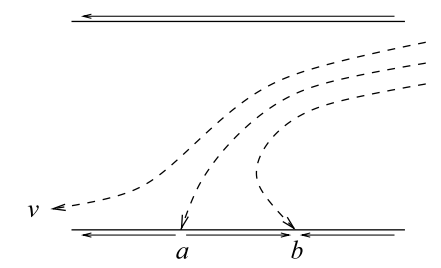

Figure 14. The flow of an (L3)-isotopy. Consider a generalized perturbed flow tree near the [-1]-chords moving upward. When one of its $b$-chords hits the flow line going to $a$ in the flow box, a 1-parameter family of perturbed flow trees becomes rigid. When the puncture moves further up, the flow goes instead to a word contributing to $v$ and stays there until the next $b$-puncture hits the flow line ending at $a$.

a generalized perturbed tree hit the $a$-flow line in the order they appear on the boundary. The formula for the differential follows.

\section{Algebraic treatment of concatenation and of homotopy of isotopies}

In this section, we first show that the DGAs of traces, after destabilization, and tame isomorphisms have certain naturality properties with respect to concatenation. Together with the results of Section 6, this lead to a proof of Theorem 1.1 as follows. Concatenation of small pieces of an isotopy yields the DGA for the full isotopy by the results of Section 6. However, this gives a DGA with an enormous number of generators. We show below, using a purely algebraic argument, that the DGA with the large number of generators is stable tame isomorphic to the DGA in the formulation of Theorem 1.1. Second, we show that the contact homology of the trace of an isotopy depends only on the chain homotopy type of the contact homology morphism induced by the isotopy. Geometrically, such a chain homotopy corresponds to a homotopy of isotopies.

7.1. Concatenation and the proof of Theorem 1.1. Let $\left(\mathcal{A}_{ \pm}, \partial_{ \pm}\right)$be a DGA with a finite set of generators $g\left(\mathcal{A}_{ \pm}\right)$and let $\varphi: \mathcal{A}_{-} \rightarrow \mathcal{A}_{+}$be a DGA morphism. Let the non-commutative algebra $\mathcal{C}_{\varphi}$ be generated by the following:

- a generator $a_{ \pm}$for each $a_{ \pm} \in g\left(\mathcal{A}_{ \pm}\right)$, with grading $\left|a_{ \pm}\right|$as in $\mathcal{A}_{ \pm}$,

- a generator $\hat{a}$ for each $a_{-} \in g\left(\mathcal{A}_{-}\right)$, with grading $|\hat{a}|=\left|a_{-}\right|+1$.

There are obvious embeddings (algebra monomorphisms) $\iota_{ \pm}: \mathcal{A}_{ \pm} \rightarrow \mathcal{C}_{\varphi}$ defined (on generators) by $\iota_{ \pm}\left(a_{ \pm}\right)=a_{ \pm}$, see Figure 15 . 


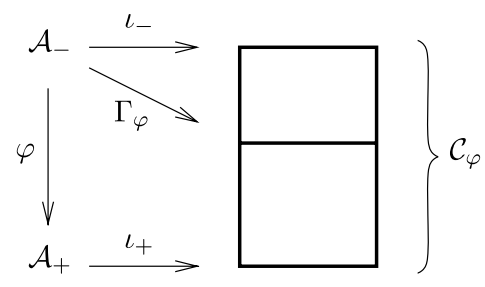

Figure 15. The mapping cone of a DGA morphism.

We define a map $\Delta_{\varphi}: \mathcal{C}_{\varphi} \rightarrow \mathcal{C}_{\varphi}$ as follows on generators

$$
\begin{aligned}
\Delta_{\varphi} a_{ \pm} & =\iota_{ \pm}\left(\partial_{ \pm} a_{ \pm}\right) \\
\Delta_{\varphi} \hat{a} & =a_{-}+\iota_{+}\left(\varphi\left(a_{-}\right)\right)+\Gamma_{\varphi}\left(\partial_{-} a_{-}\right)
\end{aligned}
$$

and extend it to all of $\mathcal{C}_{\varphi}$ by the Leibniz rule. Here $\Gamma_{\varphi}: \mathcal{A}_{-} \rightarrow \mathcal{C}_{\varphi}$ is defined as follows. On generators $a_{-}$of $\mathcal{A}_{-}$,

$$
\Gamma_{\varphi}\left(a_{-}\right)=\hat{a}
$$

and for products $u v \in \mathcal{A}_{-}$,

$$
\Gamma_{\varphi}(u v)=\iota_{-}(u) \Gamma_{\varphi}(v)+\Gamma_{\varphi}(u) \iota_{+}(\varphi(v)) .
$$

The $\left(\iota_{-}, \iota_{+} \circ \varphi\right)$-derivation $\Gamma_{\varphi}$ increases grading by 1 and consequently $\Delta_{\varphi}$ decreases grading by 1 .

Since $\partial_{ \pm}$also satisfies the Leibniz rule, it follows that $\Delta_{\varphi} \iota_{ \pm}\left(u_{ \pm}\right)=$ $\iota_{ \pm}\left(\partial_{ \pm} u_{ \pm}\right)$for all $u_{ \pm} \in \mathcal{A}_{ \pm}$. In other words, $\iota_{ \pm}$will be DGA morphisms once we show that $\left(\mathcal{C}_{\varphi}, \Delta_{\varphi}\right)$ is a DGA. To establish that we must show that $\Delta_{\varphi}^{2}=0$ and it is enough to prove this for generators of $\mathcal{C}_{\varphi}$. For generators $a_{ \pm}$this is trivial and for generators $\hat{a}$, it follows from the following lemma.

Lemma 7.1. For all $u \in \mathcal{A}_{-}$,

$$
D(u)=\iota_{-}(u)+\iota_{+}(\varphi(u))+\Delta_{\varphi} \Gamma_{\varphi}(u)+\Gamma_{\varphi}\left(\partial_{-} u\right)=0 .
$$

In particular $\Delta_{\varphi}^{2} \hat{a}=0$ for any generator $\hat{a}$.

Proof. If $a_{-}$is a generator of $\mathcal{A}_{-}$, then

$$
\begin{aligned}
D\left(a_{-}\right) & =\iota_{-}\left(a_{-}\right)+\iota_{+}\left(\varphi\left(a_{-}\right)\right)+\Delta_{\varphi} \Gamma_{\varphi}\left(a_{-}\right)+\Gamma_{\varphi}\left(\partial_{-} a_{-}\right) \\
& =a_{-}+\iota_{+}\left(\varphi\left(a_{-}\right)\right)+\Delta_{\varphi} \hat{a}+\Gamma_{\varphi}\left(\partial_{-} a_{-}\right)=0
\end{aligned}
$$


by definition of $\Delta_{\varphi} \hat{a}$. If $D(u)=D(v)=0$, then

$$
\begin{aligned}
D(u v)= & \iota_{-}(u v)+\iota_{+}(\varphi(u v))+\Delta_{\varphi} \Gamma_{\varphi}(u v)+\Gamma_{\varphi}(\partial(u v)) \\
= & \iota_{-}(u) \iota_{-}(v)+\iota_{+}(\varphi(u)) \iota_{+}(\varphi(v))+\Delta_{\varphi}\left(\iota_{-}(u) \Gamma_{\varphi}(v)\right. \\
& \left.+\Gamma_{\varphi}(u) \iota_{+}(\varphi(v))\right)+\Gamma_{\varphi}\left(\partial_{-} u \cdot v+u \cdot \partial_{-} v\right) \\
= & \iota_{-}(u) \iota_{-}(v)+\iota_{+}(\varphi(u)) \iota_{+}(\varphi(v)) \\
& +\Delta_{\varphi}\left(\iota_{-}(u)\right) \cdot \Gamma_{\varphi}(v)+\iota_{-}(u) \cdot \Delta_{\varphi} \Gamma_{\varphi}(v)+\Delta_{\varphi} \Gamma_{\varphi}(u) \cdot \iota_{+}(\varphi(v)) \\
& +\Gamma_{\varphi}(u) \cdot \Delta_{\varphi} \iota_{+}(\varphi(v))+\iota_{-}\left(\partial_{-} u\right) \Gamma_{\varphi}(v)+\Gamma_{\varphi}\left(\partial_{-} u\right) \iota_{+}(\varphi(v)) \\
& +\iota_{-}(u) \Gamma_{\varphi}(\partial v)+\Gamma_{\varphi}(u) \iota_{+}(\varphi(\partial v)) \\
= & \left(\iota_{+}(\varphi(u))+\Delta_{\varphi} \Gamma_{\varphi}(u)+\Gamma_{\varphi}\left(\partial_{-} u\right)\right) j(\varphi(v))+\iota_{-}(u)\left(\iota_{-}(v)\right. \\
& \left.+\Delta_{\varphi} \Gamma_{\varphi}(v)+\Gamma_{\varphi}\left(\partial_{-} v\right)\right) \\
= & \iota_{-}(u) \iota_{+}(\varphi(v))+\iota_{-}(u) \iota_{+}(\varphi(v))=0 .
\end{aligned}
$$

For the second statement, note that $\Delta_{\varphi}^{2} \hat{a}=D\left(\partial_{-} a\right)$. $\mathcal{A}_{+}$.

We call the DGA $\left(\mathcal{C}_{\varphi}, \Delta_{\varphi}\right)$ the mapping cone of the chain map $\varphi: \mathcal{A}_{-} \rightarrow$

The proof of the main theorem of this section relies on the following result which is often used in the subject of contact homology. For a proof see $[\mathbf{3}]$.

Theorem 7.2. Let $\mathcal{A}=T\left(q_{1}, q_{2}, \ldots, q_{m}, a, b\right)$ and $\mathcal{A}^{\prime}=T\left(q_{1}, q_{2}, \ldots, q_{m}\right)$ be $D G A s$ freely generated by the indicated generators with differentials $\partial$ and $\partial^{\prime}$, respectively, which decrease grading by one and which satisfy the Leibniz rule. Assume that $\mathcal{A}$ and $\mathcal{A}^{\prime}$ come equipped with height filtrations, that is, that there exists an ordering of the generators $c \in\left\{q_{1}, \ldots, q_{m}, a, b\right\}$ of $\mathcal{A}$ with the following property. For each $c$, the expression $\partial c\left(\partial^{\prime} c\right)$ is a polynomial of other generators of $\mathcal{A}\left(\right.$ of $\left.\mathcal{A}^{\prime}\right)$ in which all generators are of smaller height than c. Assume that $\partial a=b+v$. Define the projection

$$
\tau: \mathcal{A} \rightarrow \mathcal{A}^{\prime}
$$

by $\tau\left(q_{j}\right)=q_{j}, 1 \leq j \leq m, \tau(a)=0$, and $\tau(b)=v$. Extend $\tau$ to a grading preserving algebra homomorphism and suppose it is a chain map. Then, the $D G A s(\mathcal{A}, \partial)$ and $\left(\mathcal{A}^{\prime}, \partial^{\prime}\right)$ are stable tame isomorphic. In particular, they have isomorphic homologies.

Let now $\left(\mathcal{A}_{1}, \partial_{1}\right),\left(\mathcal{A}_{2}, \partial_{2}\right)$, and $\left(\mathcal{A}_{3}, \partial_{3}\right)$ be filtered DGAs with $\alpha: \mathcal{A}_{1} \rightarrow$ $\mathcal{A}_{2}$ and $\beta: \mathcal{A}_{2} \rightarrow \mathcal{A}_{3}$ DGA morphisms between them (see Figure 16). Let $\left(\mathcal{C}_{\alpha}, \Delta_{\alpha}\right)$ be the mapping cone of $\alpha$ and $\left(\mathcal{C}_{\beta}, \Delta_{\beta}\right)$ be the mapping cone of $\beta$. Define the algebra $\mathcal{B}$ by taking the disjoint union of $\mathcal{C}_{\alpha}$ and $\mathcal{C}_{\beta}$ and identifying for each generator $b$ of $\mathcal{A}_{2}$, the corresponding generator $b \in \mathcal{C}_{\alpha}$ with $b \in \mathcal{C}_{\beta}$. This is the algebraic version of concatenation and $\Delta_{\alpha}$ and $\Delta_{\beta}$ 


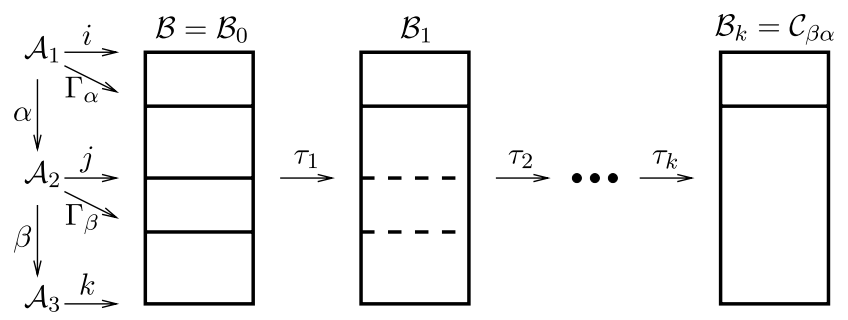

Figure 16. Concatenating mapping cones.

define a differential $\Delta$ on $\mathcal{B}$. Note that the inclusions of $\mathcal{A}_{j} \rightarrow \mathcal{B}, j=1,2,3$, are DGA morphisms.

Lemma 7.3. The DGA $(\mathcal{B}, \Delta)$ is stable tame isomorphic to the mapping cone $\left(\mathcal{C}_{\beta \alpha}, \Delta_{\beta \alpha}\right)$ of the chain map $\beta \circ \alpha: \mathcal{A}_{1} \rightarrow \mathcal{A}_{3}$.

Proof. We remove pairs of generators $(b, \hat{b})$ for $b \in \mathcal{A}_{2}$ inductively. Let the generators of $\mathcal{A}_{2}$ be arranged by height from $b_{1}$ (the longest) to $b_{k}$ (the shortest). We eliminate $b_{1}$ first, then the second longest generator $b_{2}$, and so on until we reach $b_{k}$. Starting from $\mathcal{B}=\mathcal{B}_{0}$, we obtain the DGAs $\mathcal{B}_{i}$ $(i=0,1, \ldots, k)$ that are generated by $a$ and $\hat{a}$ for each generator $a \in \mathcal{A}_{1}$, $c$ for each generator $c \in \mathcal{A}_{3}$, and by $b_{i+1}, \hat{b}_{i+1}, \ldots, b_{k}, \hat{b}_{k}$. We may assume that in each $\mathcal{B}_{i}$, the heights of the $\hat{a}$ generators are all greater than those of the $b$ and $\hat{b}$ generators.

Starting from $\Delta_{0}=\Delta$, we define differentials $\Delta_{i}$ on $\mathcal{B}_{i}$ recursively. If $\Delta_{i-1} \hat{b}_{i}=b_{i}+v_{i}$, then we define the projection $\tau_{i}: \mathcal{B}_{i-1} \rightarrow \mathcal{B}_{i}$ as in Theorem 7.2. (We remark that $\Delta_{i-1} \hat{b}_{i}$ will always have that form, in fact with $v_{i}=\beta\left(b_{i}\right)+\Gamma_{\beta}\left(\partial_{2} b_{i}\right)$, since we eliminate in the order of decreasing height; the interesting part of this definition is $\Delta_{i} \hat{a}$ for $a \in \mathcal{A}_{1}$.) For any generator $c$ of $\mathcal{B}_{i}$, we define $\Delta_{i} c=\tau_{i}\left(\Delta_{i-1} c\right)$. Then $\tau_{i}$ is a DGA morphism and Theorem 7.2 implies that all $\mathcal{B}_{i}$ are stable tame isomorphic.

To finish the proof we use the following: for all $b \in \mathcal{A}_{2} \subset \mathcal{B}$,

$$
\tau_{k} \circ \tau_{k-1} \circ \cdots \circ \tau_{1}(b)=\beta(b) .
$$

To see this, note that

$$
\tau_{k} \circ \tau_{k-1} \circ \cdots \circ \tau_{1}\left(b_{i}\right)=\tau_{k} \circ \cdots \circ \tau_{i}\left(b_{i}\right)=\tau_{k} \circ \cdots \circ \tau_{i+1}\left(\beta\left(b_{i}\right)+\Gamma_{\beta}\left(\partial_{2} b_{i}\right)\right) .
$$

Here, each monomial of $\Gamma_{\beta}\left(\partial_{2} b_{i}\right)$ contains a $\hat{b}_{j}$-factor for some $i+1 \leq j \leq k$, thus one of the remaining projections will annihilate it, and $\beta\left(b_{i}\right)$ is in $\mathcal{A}_{3}$ and is therefore fixed by all projections.

We claim that $\left(\mathcal{B}_{k}, \Delta_{k}\right)$ is isomorphic to the mapping cone $\left(\mathcal{C}_{\beta \alpha}, \Delta_{\beta \alpha}\right)$. Their sets of generators agree and the differentials for generators $a \in \mathcal{A}_{1}$ 
and $c \in \mathcal{A}_{3}$ are obviously the same. For any generator $a \in \mathcal{A}_{1}$,

$$
\begin{aligned}
\Delta_{k} \hat{a} & =\Delta_{k} \tau_{k} \circ \tau_{k-1} \circ \cdots \circ \tau_{1}(\hat{a}) \\
& =\tau_{k} \circ \tau_{k-1} \circ \cdots \circ \tau_{1}\left(\Delta_{0} \hat{a}\right) \\
& =\tau_{k} \circ \tau_{k-1} \circ \cdots \circ \tau_{1}\left(a+\alpha(a)+\Gamma_{\alpha}\left(\partial_{1} a\right)\right) \\
& =a+\tau_{k} \circ \tau_{k-1} \circ \cdots \circ \tau_{1}(\alpha(a))+\tau_{k} \circ \tau_{k-1} \circ \cdots \circ \tau_{1}\left(\Gamma_{\alpha}\left(\partial_{1} a\right)\right) \\
& \left.=a+\beta(\alpha(a))+\Gamma_{\beta \circ \alpha}\left(\partial_{1} a\right)\right) .
\end{aligned}
$$

To see that the last equality holds, note that each summand of $\Gamma_{\alpha}\left(\partial_{1} a\right)$ is a three-fold product of an element of $\mathcal{A}_{1}$ (possibly 1), an $\hat{a}$-generator (these are all fixed by projections), and the $\alpha$-image of an element of $\mathcal{A}_{1}$; note that projections are algebra homomorphisms and use (7.2).

Proof of Theorem 1.1. Subdivide the isotopy in sufficiently small pieces so that each piece contains at most one move, see Section 6 for a description of moves, and so that the isotopy of each piece without move is sufficiently close to constant. Then Lemma 6.1 implies that the DGA of each piece without move is isomorphic to the DGA of the trace of the constant isotopy and Corollary 6.2 gives the DGA for such pieces. Lemmas $6.3-6.8$ give the DGAs for pieces with moves. Concatenating all pieces gives a DGA with many generators corresponding to the full isotopy. Lemma 7.3 shows that this many-generator DGA is stable tame isomorphic to the DGA in the formulation of the theorem.

7.2. Chain homotopy. Consider two DGAs $\left(\mathcal{A}_{+}, \partial_{+}\right)$and $\left(\mathcal{A}_{-}, \partial_{-}\right)$. Let $\psi, \phi: \mathcal{A}_{+} \rightarrow \mathcal{A}_{-}$be chain maps and let $K: \mathcal{A}_{+} \rightarrow \mathcal{A}_{-}$be a chain homotopy between them. That is,

$$
\phi+\psi=K \circ \partial_{+}+\partial_{-} \circ K
$$

Consider the mapping cone DGAs $\left(\mathcal{C}_{\phi}, \Delta_{\phi}\right)$ and $\left(\mathcal{C}_{\psi}, \Delta_{\psi}\right)$. Define the map $\Gamma_{K}: \mathcal{A}_{+} \rightarrow \mathcal{C}_{\psi}$ to equal zero on constants and on linear monomials and by the following expression for monomials $b_{1} \cdots b_{r}$ of length $r \geq 2$

$\Gamma_{K}\left(b_{1} \cdots b_{r}\right)=\hat{b}_{1} K\left(b_{2} \cdots b_{r}\right)+b_{1} \hat{b}_{2} K\left(b_{3} \cdots b_{r}\right)+\cdots+b_{1} \cdots b_{r-2} \hat{b}_{r-1} K\left(b_{r}\right)$.

Lemma 7.4. The algebra map $F:\left(\mathcal{C}_{\phi}, \Delta_{\phi}\right) \rightarrow\left(\mathcal{C}_{\psi}, \Delta_{\psi}\right)$ defined on generators as follows

$$
\begin{aligned}
& F(c)=c, \quad c \in \mathcal{A}_{+}, \\
& F(v)=v, \quad v \in \mathcal{A}_{-}, \\
& F(\hat{c})=\hat{c}+K(c)+\Gamma_{K}\left(\partial_{+} c\right), \quad c \in \hat{\mathcal{A}}_{+},
\end{aligned}
$$

is a (tame) chain isomorphism. That is,

$$
F \circ \Delta_{\phi}=\Delta_{\psi} \circ F \text {. }
$$


Proof. For simpler notation, we write $\mathcal{C}_{\phi}=\mathcal{A}=\mathcal{C}_{\psi}$ and consider this as one algebra with two differentials and in order to facilitate computations, we introduce the following notation. Let $H: \mathcal{A}_{+} \rightarrow \mathcal{A}$ and $\theta: \mathcal{A}_{+} \rightarrow A$ be maps. Then define the map $\Omega_{\theta}^{H}: \mathcal{A}_{+} \rightarrow \mathcal{A}$ as follows on monomials

$\Omega_{\theta}^{H}\left(b_{1} \cdots b_{r}\right)=H\left(b_{1}\right) \theta\left(b_{2} \cdots b_{r}\right)+b_{1} H\left(b_{2}\right) \theta\left(b_{3} \cdots b_{r}\right)+\cdots+b_{1} \cdots b_{r-1} H\left(b_{r}\right)$.

We compute

$$
\begin{aligned}
F\left(\Delta_{\phi}(\hat{c})\right) & =F\left(c+\phi(c)+\Gamma_{\phi}\left(\partial_{+} c\right)\right) \\
& =c+\phi(c)+\Gamma_{\phi}\left(\partial_{+} c\right)+\Omega_{\phi}^{K}\left(\partial_{+} c\right)+\Omega_{\phi}^{\Gamma_{K} \circ \partial_{+}}\left(\partial_{+} c\right),
\end{aligned}
$$

and

$$
\begin{aligned}
\Delta_{\psi}(F(\hat{c})) & =\Delta_{\psi}\left(\hat{c}+K(c)+\Gamma_{K}\left(\partial_{+} c\right)\right) \\
& =c+\psi(c)+\Gamma_{\psi}\left(\partial_{+} c\right)+\partial_{-}(K(c))+\Delta_{\psi}\left(\Gamma_{K}\left(\partial_{+} c\right)\right) .
\end{aligned}
$$

We note that the monomials in (7.3) and (7.4) are of two types: monomials

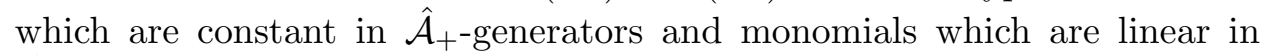
$\hat{\mathcal{A}}_{+}$-generators. We first show that the monomials of the former kind cancel between the two equations.

The contribution from (7.3) to monomials of the first kind is

$$
c+\phi(c)+\Omega_{\phi}^{K}\left(\partial_{+} c\right),
$$

and the contribution from (7.4) is

$$
c+\psi(c)+\partial_{-}(K(c))+\Delta_{\psi}\left(\Gamma_{K}\left(\partial_{+} c\right)\right)_{0},
$$

where $w_{0}$ denotes the term in an element which is constant in the $\hat{\mathcal{A}}_{+}$generators. Thus, if we show that

$$
\Omega_{\phi}^{K}\left(\partial_{+} c\right)+\Delta_{\psi}\left(\Gamma_{K}\left(\partial_{+} c\right)\right)_{0}=K\left(\partial_{+} c\right),
$$

then it follows that monomials of the first type cancel. Now, if $b_{1} \cdots b_{r}$ is a monomial in $\partial_{+} c$, then the contribution from this monomial to the left-hand side of (7.5), which is non-constant in the $\mathcal{A}_{+}$-generators, vanishes for the following reason. Terms arising from $\Omega_{\phi}^{K}$ have the form

$$
b_{1} \cdots b_{s} K\left(b_{s+1}\right) \phi\left(b_{s+2}\right) \cdots \phi\left(b_{r}\right)
$$

and are canceled by terms in $\Delta_{\psi}\left(b_{1} \cdots \hat{b}_{s} K\left(b_{s+1}\right) \phi\left(b_{s+2}\right) \cdots \phi\left(b_{r}\right)\right)$ corresponding to the $b_{s}$-term in $\Delta_{\psi}\left(\hat{b}_{s}\right)$. Remaining terms in $\Delta_{\psi}\left(b_{1} \ldots \hat{b}_{s}\right.$ $\left.K\left(b_{s+1} \ldots b_{r}\right)\right)$ corresponding to the $b_{s}$-term in $\Delta_{\psi}\left(\hat{b}_{s}\right)$ cancel with terms in $\Delta_{\psi}\left(b_{1} \ldots b_{s} \hat{b}_{s+1} K\left(b_{s+2} \ldots b_{r}\right)\right)$ corresponding to the $\psi\left(b_{s+1}\right)$-term in $\Delta_{\psi}\left(\hat{b}_{s+1}\right)$. Thus if $b_{1} \ldots b_{r}$ is any monomial in $\partial_{+} c$, then its contribution to the left-hand side of (7.5) is exactly

$K\left(b_{1}\right) \phi\left(b_{2}\right) \cdots \phi\left(b_{r}\right)+\psi\left(b_{1}\right) K\left(b_{2}\right) \phi\left(b_{3}\right) \cdots \phi\left(b_{r}\right)+\cdots \psi\left(b_{1}\right) \cdots \psi\left(b_{r-1}\right) K\left(b_{r}\right)$ and (7.5) follows. 
We next consider monomials linear in $\hat{\mathcal{A}}_{+}$-generators contributing to (7.3) and (7.4). To this end, we define the maps $A, B, C, D: \mathcal{A}_{+} \rightarrow \mathcal{A}$ as follows on monomials.

$$
\begin{aligned}
A\left(b_{1} \cdots b_{s}\right)= & \Gamma_{\psi}\left(\partial_{+} b_{1}\right) K\left(b_{2} \cdots b_{s}\right)+b_{1} \Gamma_{\psi}\left(\partial_{+} b_{2}\right) K\left(b_{3} \cdots b_{s}\right) \\
& +\cdots+b_{1} \cdots b_{s-2} \Gamma_{\psi}\left(b_{s-1}\right) K\left(b_{s}\right)
\end{aligned}
$$

if $s \geq 2$ and 0 otherwise.

$$
\begin{aligned}
B\left(b_{1} \cdots b_{s}\right)= & \hat{b}_{1} K\left(\partial_{+}\left(b_{2} \cdots b_{s}\right)\right)+b_{1} \hat{b}_{2} K\left(\partial_{+}\left(b_{3} \cdots b_{s}\right)\right) \\
& +\cdots+b_{1} \cdots b_{s-2} \hat{b}_{s-1} K\left(\partial_{+}\left(b_{s}\right)\right)
\end{aligned}
$$

if $s \geq 2$ and 0 otherwise.

$$
\begin{aligned}
C\left(b_{1} \cdots b_{s}\right)= & \left(\partial_{+} b_{1}\right) \hat{b}_{2} K\left(b_{3} \cdots b_{s}\right)+\partial_{+}\left(b_{1} b_{2}\right) \hat{b}_{3} K\left(\left(b_{4} \cdots b_{s}\right)\right) \\
& +\cdots+\partial\left(b_{1} \cdots b_{s-2}\right) \hat{b}_{s-1} K\left(b_{s}\right),
\end{aligned}
$$

if $s \geq 2$ and 0 otherwise.

$$
\begin{aligned}
D\left(b_{1} \cdots b_{s}\right)= & \hat{b}_{1} \partial_{-}\left(K\left(b_{2} \cdots b_{s}\right)\right)+b_{1} \hat{b}_{2} \partial_{-}\left(K\left(b_{3} \cdots b_{s}\right)\right) \\
& +\cdots+b_{1} \cdots b_{s-2} \hat{b}_{s-1} \partial_{-}\left(K\left(b_{s}\right)\right),
\end{aligned}
$$

if $s \geq 2$ and 0 otherwise.

We have

$$
0=\Gamma_{K}\left(\partial_{+} \partial_{+} c\right)=\Omega^{\Gamma_{K} \circ \partial_{+}}\left(\partial_{+} c\right)+A\left(\partial_{+} c\right)+B\left(\partial_{+} c\right)+C\left(\partial_{+} c\right) .
$$

(To see this, one subdivides contributing monomials as follows. Let $b_{1} \cdots b_{s}$ be a monomial in $\partial_{+} c$. The first term corresponds to the $\hat{\mathcal{A}}_{+}$-variable and the $K$-variable both located in a $\partial b_{j}$-monomial for some $j$. The second

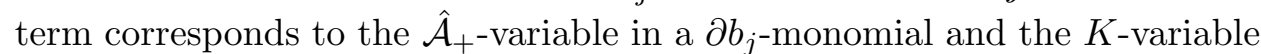
outside. The third term corresponds to the $\hat{\mathcal{A}}_{+}$-variable outside a $\partial_{+} b_{j}$ monomial and the variable on which the $\partial_{+}$-operator acts being on the right of the $\hat{\mathcal{A}}_{+}$-generator. The fourth term corresponds to the $\hat{\mathcal{A}}_{+}$-variable outside a $\partial_{+} b_{j}$-monomial and the variable on which the $\partial_{+}$-operator acts being on the left of the $\hat{\mathcal{A}}_{+- \text {generator.) }}$

Similarly, we have

$$
\Delta_{\psi}\left(\Gamma_{K}\left(\partial_{+} c\right)\right)_{1}=A\left(\partial_{+} c\right)+C\left(\partial_{+} c\right)+D\left(\partial_{+} c\right),
$$

where $w_{1}$ denotes the term of an element which is linear in the $\hat{\mathcal{A}}_{+^{-}}$ generators. Consequently, the contribution of monomials of the second kind to the sum of (7.3) and (7.4) is

$$
\begin{gathered}
\Gamma_{\phi}\left(\partial_{+} c\right)+\Gamma_{\psi}\left(\partial_{+} c\right)+\Omega^{\Gamma_{K} \circ \partial_{+}}\left(\partial_{+} c\right)+\Delta_{\psi}\left(\Gamma_{K}\left(\partial_{+} c\right)\right)_{1} \\
\quad=\Gamma_{\phi}\left(\partial_{+} c\right)+\Gamma_{\psi}\left(\partial_{+} c\right)+B\left(\partial_{+} c\right)+D\left(\partial_{+} c\right)=0 .
\end{gathered}
$$

The lemma follows. 


\section{Examples}

The results of this paper allow us to construct many interesting Legendrian submanifolds. To illustrate this, we shall apply Theorem 1.1 to some of the loops of Legendrian knots discussed in [15]. In particular, we will concentrate on augmentations of the contact homology of the resulting Legendrian tori. Our examples give a proof of Theorem 1.2.

In any situation when the Legendrian $L \subset J^{1}(\mathbb{R})$, of Maslov class $r=0$, only has Reeb chords of non-negative grading (and $L$ is the base point of a closed loop that gives rise to the Legendrian torus $\Lambda$ ), the following observation applies. The "long chords" (hat variables) of $\Lambda$ have positive grading so they cannot be augmented. We have to select index 0 chords $b$ of $L$ ("short chords") on which the augmentation should take a non-zero value. Grading 1 short chords impose the same relations on these as when searching for augmentations of $L$ itself. So augmentations of $\Lambda$ are in fact also augmentations of $L$. But there are also relations imposed by the grading 1 long chords. Because of the absence of negative grading chords, these are of the form $\Delta \hat{b}=b+\phi(b)$, where $\phi$ is the monodromy of our loop. Hence, an augmentation of $L$ will be an augmentation of $\Lambda$ if and only if it is invariant under $\phi$.

In [15], a natural loop is described in the space of braid-positive Legendrian knots. The $(p, 2)$ torus knots are its simplest special case, when conjugating a single crossing from one end of the braid to the other already results in a closed loop. We will examine the cases $p=3$ and $p=7$.

Proof of Theorem 1.2 (a). The diagram of the loop for $p=3$ is reprinted in Figure 17. We recall from [15, Section 5] that, after restoring the original labels, the monodromy $\phi$ of this loop acts on the index 0 variables $b_{1}, b_{2}$,

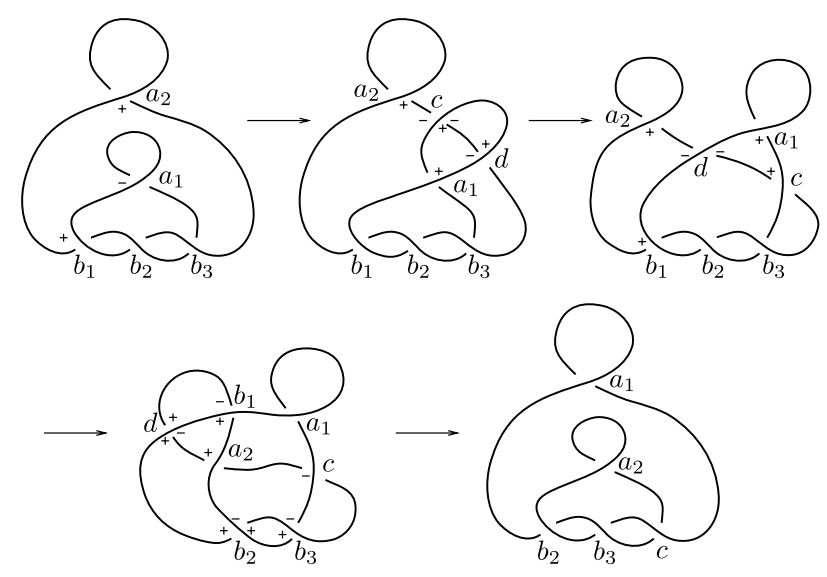

Figure 17. A loop of Legendrian trefoil knots. 
and $b_{3}$ as follows:

$$
\phi\left(b_{1}\right)=1+b_{2} b_{3} ; \quad \phi\left(b_{2}\right)=b_{1} ; \quad \phi\left(b_{3}\right)=b_{2} .
$$

The crossings $a_{1}$ and $a_{2}$ are of index 1 and they are not cycles, so for the time being we only concern ourselves with their boundaries (see, for example, [15, Example 2.14]):

$$
\partial a_{1}=1+b_{1}+b_{3}+b_{1} b_{2} b_{3} ; \quad \partial a_{2}=b_{2}+b_{1} b_{2}+b_{2} b_{3}+b_{2} b_{3} b_{1} b_{2} .
$$

The other three relations that determine the index 0 part $\mathrm{CH}_{0}$ of the contact homology of the corresponding torus are

$$
\Delta \hat{b}_{1}=b_{1}+1+b_{2} b_{3} ; \quad \Delta \hat{b}_{2}=b_{2}+b_{1} ; \quad \Delta \hat{b}_{3}=b_{3}+b_{2}
$$

The last two relations in (8.1) imply that $\mathrm{CH}_{0}$ has a single generator. We denote it $b$. It is subject to the relations

$$
1+b^{3}=0 ; \quad b+b^{4}=0 ; \quad 1+b+b^{2}=0 .
$$

The first two of these follow from the third by multiplication with $b+1$ and $b(b+1)$, respectively. (Recall that we work over $\mathbb{Z}_{2}$.) Therefore,

$$
C H_{0} \cong \mathbb{Z}_{2}[b] /\left\langle 1+b+b^{2}=0\right\rangle .
$$

Thus $\mathrm{CH}_{0}$ is non-trivial. On the other hand, because neither 0 nor 1 is a root of the relation, this contact homology cannot be augmented. (Indeed, the five augmentations of the trefoil are permuted in a single cycle by $\phi$, so none of them is fixed.)

Proof of Theorem 1.2 (b). Let $p=7$ for the knots considered above. For convenience, we introduce terminology that applies for any odd number $p$. The loop is the same as in Figure 17 except that there are $p-3$ more index 0 crossings $b_{4}, \ldots, b_{p}$ that, just like $b_{2}$ and $b_{3}$, are essentially unaffected by the monodromy (they simply get re-labeled at the end).

They do however influence $\partial a_{1}$ and $\partial a_{2}$ as follows:

$$
\partial a_{1}=1+B_{11} ; \quad \partial a_{2}=1+B_{22}+B_{21} B_{12} .
$$

Here, the polynomials $B_{i j}$ are natural generalizations of the expressions $B_{11}=b_{1}+b_{3}+b_{1} b_{2} b_{3}, B_{12}=1+b_{2} b_{3}, B_{21}=1+b_{1} b_{2}$, and $B_{22}=b_{2}$ in the $p=3$ case; see $[\mathbf{1 5}$, Section 6$]$ for more details. In particular when $p=7$, 
we obtain

$$
\begin{aligned}
\Delta a_{1}=\partial a_{1}= & 1+b_{1}+b_{3}+b_{5}+b_{7}+b_{1} b_{2} b_{3}+b_{1} b_{2} b_{5}+b_{1} b_{2} b_{7} \\
& +b_{1} b_{4} b_{5}+b_{1} b_{4} b_{7}+b_{1} b_{6} b_{7}+b_{3} b_{4} b_{5}+b_{3} b_{4} b_{7}+b_{3} b_{6} b_{7} \\
& +b_{5} b_{6} b_{7}+b_{1} b_{2} b_{3} b_{4} b_{5}+b_{1} b_{2} b_{3} b_{4} b_{7}+b_{1} b_{2} b_{3} b_{6} b_{7}+b_{1} b_{2} b_{5} b_{6} b_{7} \\
& +b_{1} b_{4} b_{5} b_{6} b_{7}+b_{3} b_{4} b_{5} b_{6} b_{7}+b_{1} b_{2} b_{3} b_{4} b_{5} b_{6} b_{7} \\
\Delta a_{2}=\partial a_{2}= & +b_{2}+b_{4}+b_{6}+b_{2} b_{3} b_{4}+b_{2} b_{3} b_{6}+b_{2} b_{5} b_{6}+b_{4} b_{5} b_{6} \\
& +b_{2} b_{3} b_{4} b_{5} b_{6}+\left(1+b_{2} b_{3}+b_{2} b_{5}+b_{2} b_{7}+b_{4} b_{5}+b_{4} b_{7}+b_{6} b_{7}\right. \\
& +b_{2} b_{3} b_{4} b_{5}+b_{2} b_{3} b_{4} b_{7}+b_{2} b_{3} b_{6} b_{7}+b_{2} b_{5} b_{6} b_{7}+b_{4} b_{5} b_{6} b_{7} \\
& \left.+b_{2} b_{3} b_{4} b_{5} b_{6} b_{7}\right) \cdot\left(1+b_{1} b_{2}+b_{1} b_{4}+b_{1} b_{6}+b_{3} b_{4}+b_{3} b_{6}+b_{5} b_{6}\right. \\
& +b_{1} b_{2} b_{3} b_{4}+b_{1} b_{2} b_{3} b_{6}+b_{1} b_{2} b_{5} b_{6}+b_{1} b_{4} b_{5} b_{6}+b_{3} b_{4} b_{5} b_{6} \\
& \left.+b_{1} b_{2} b_{3} b_{4} b_{5} b_{6}\right) .
\end{aligned}
$$

These formulas are special cases of $\left[\mathbf{1 5}\right.$, Theorem 6.7]. The relations $\Delta \hat{b}_{2}=$ $b_{2}+b_{1}=0, \ldots, \Delta \hat{b}_{p}=b_{p}+b_{p-1}=0$ reduce $C H_{0}$ of this torus to a singlegenerator algebra. If $b$ denotes a generator of $C H_{0}$ then, when $p=7$, the previous two formulas impose the relations

$$
1+b^{7}=0 \quad \text { and } \quad 1+b+b^{5}+\left(1+b^{4}+b^{6}\right)^{2}=b+b^{5}+b^{8}+b^{12}=0 .
$$

The second of these is equal to $b+b^{5}$ times the first. For a general $p$, identifying the grading 0 generators leads to the reductions

$$
B_{11} \mapsto Q_{p}(b), \quad B_{12}, B_{21} \mapsto Q_{p-1}(b), \quad B_{22} \mapsto Q_{p-2}(b),
$$

where the polynomials $Q_{k}$ are defined by setting $Q_{-1}(b)=0, Q_{0}(b)=1$ and then applying the recursion $Q_{k}(b)=b Q_{k-1}(b)+Q_{k-2}(b)$. They are also characterized by the formula

$$
\left[\begin{array}{ll}
b & 1 \\
1 & 0
\end{array}\right]^{k}=\left[\begin{array}{cc}
Q_{k}(b) & Q_{k-1}(b) \\
Q_{k-1}(b) & Q_{k-2}(b)
\end{array}\right] .
$$

(This follows by a straightforward induction argument, and explicit formulas for the coefficients can be obtained from Pascal's triangle). In particular, because the determinant of the above matrix is $1, \Delta a_{2}=\partial a_{2}=1+B_{22}+$ $B_{21} B_{12}$ reduces to $1+Q_{p-2}(b)+Q_{p-1}(b)^{2}=Q_{p-2}(b)+Q_{p}(b) Q_{p-2}(b)=(1+$ $\left.Q_{p}(b)\right) Q_{p-2}(b)$. Here of course, $1+Q_{p}(b)$ is just the reduction of $\Delta a_{1}=\partial a_{1}$. Thus so far, we obtained only one relation, $1+Q_{p}(b)=0$, for the single generator $b$ of $\mathrm{CH}_{0}$.

The other relation, again for $p=7$, comes from

$$
\begin{aligned}
\Delta \hat{b}_{1}= & b_{1}+1+b_{2} b_{3}+b_{2} b_{5}+b_{2} b_{7}+b_{4} b_{5}+b_{4} b_{7}+b_{6} b_{7}+b_{2} b_{3} b_{4} b_{5} \\
& +b_{2} b_{3} b_{4} b_{7}+b_{2} b_{3} b_{6} b_{7}+b_{2} b_{5} b_{6} b_{7}+b_{4} b_{5} b_{6} b_{7}+b_{2} b_{3} b_{4} b_{5} b_{6} b_{7},
\end{aligned}
$$

which simplifies to

$$
1+b+b^{4}+b^{6}=0 .
$$


(For general $p, \Delta \hat{b}_{1}=b_{1}+B_{21}$, which reduces to $b+Q_{p-1}(b)$. This follows from Theorem 1.1 and [15, Proposition 8.2].) The Euclidean algorithm shows that the greatest common divisor of this and $1+b^{7}$ is $1+b^{2}+b^{3}+b^{4}$. (Indeed, $\left(1+b+b^{2}\right)\left(1+b^{2}+b^{3}+b^{4}\right)=1+b+b^{4}+b^{6}$ and $\left(1+b^{2}+b^{3}\right)$ $\left(1+b^{2}+b^{3}+b^{4}\right)=1+b^{7}$.) Thus in this case,

$$
C H_{0} \cong \mathbb{Z}_{2}[b] /\left\langle 1+b^{2}+b^{3}+b^{4}=0\right\rangle .
$$

Setting $b=1$ defines the only augmentation; this corresponds to the fact that $\varepsilon\left(b_{1}\right)=\cdots=\varepsilon\left(b_{7}\right)=1$ is an invariant augmentation of the $(7,2)$ torus knot. (Other, more typical augmentations of the knot are not invariant under the monodromy of the loop: 81 of them form nine 9-cycles, and there is a 3-cycle too.) With this augmentation of the torus, the linearized differential $\tilde{\Delta}$ takes the following form:

$$
\begin{aligned}
& \tilde{\Delta} b_{1}=\cdots=\tilde{\Delta} b_{7}=0 ; \quad \tilde{\Delta} a_{1}=b_{1}+b_{4}+b_{7}, \quad \tilde{\Delta} a_{2}=0 \\
& \tilde{\Delta} \hat{b}_{1}=b_{1}+b_{3}+b_{6}, \quad \tilde{\Delta} \hat{b}_{2}=b_{1}+b_{2}, \ldots, \tilde{\Delta} \hat{b}_{7}=b_{6}+b_{7} \\
& \tilde{\Delta} \hat{a}_{1}=a_{1}+a_{2}+\hat{b}_{1}+\hat{b}_{4}+\hat{b}_{7}, \quad \tilde{\Delta} \hat{a}_{2}=0 .
\end{aligned}
$$

Here, (8.3) and (8.4) follow directly from the formulas already given, but (8.5) needs explanation. First, we need to understand $\phi\left(a_{1}\right)$ and $\phi\left(a_{2}\right)$. The key to this is [15, Remark 3.4] and the second diagram in Figure 17. Note that there exist no admissible disks there with a positive corner at $a_{1}$ and a negative corner at the newly created $c$. Thus the image of $a_{1}$ is itself in this step, and it is not affected later either, except for re-labeling to $\phi\left(a_{1}\right)=a_{2}$ at the end. There are, however, admissible disks from $a_{2}$ to $c$. With their contributions, the image of $a_{2}$ is $a_{2}+B_{21} d$. The two triangle moves that follow affect $d$, namely $d \mapsto d+a_{1} c \mapsto d+b_{1} a_{2}+a_{1} c$. Then at the last move before re-labeling, $d \mapsto 0$ and $b_{1} \mapsto B_{21}^{\prime}$. Here, $B_{21}^{\prime}$ refers to the braid with crossings labeled $b_{2}, \ldots, b_{p}, c$. So far, the image of $a_{2}$ is $a_{2}+B_{21}\left(B_{21}^{\prime} a_{2}+a_{1} c\right)$. This gets re-labeled to $\phi\left(a_{2}\right)=a_{1}+B_{12}\left(B_{21} a_{1}+a_{2} b_{p}\right)$.

So we have

$$
\Delta \hat{a}_{1}=a_{1}+a_{2}+\Gamma_{\phi}\left(\partial a_{1}\right)
$$

and

$$
\Delta \hat{a}_{2}=a_{2}+a_{1}+B_{12} B_{21} a_{1}+B_{12} a_{2} b_{p}+\Gamma_{\phi}\left(\partial a_{2}\right) .
$$

All monomials in these expressions have a single grading 1 factor and several augmented grading 0 factors. To linearize them, we just have to count the number of times each grading 1 variable appears. When $p=7$, we have already checked that $B_{12}$ and $B_{21}$ are sums of an odd number of terms (13, to be exact). To compute the contributions from the $\Gamma_{\phi}\left(\partial a_{i}\right)$ terms, note that it is essentially the same task as the linearization of $\Delta a_{i}=\partial a_{i}$ : each 
monomial contributes the sum of its terms to the latter, and the sum of the hat equivalents of its terms to the former.

Now it is straightforward to compute that the homology of this complex has rank 1 in gradings 1 and 2 and rank 0 everywhere else. This is identical to the linearized contact homology of the standard torus that is the trace of the constant isotopy of the unknot. (The latter has single generators in gradings 1 and 2 and a trivial differential, hence 0 is its only augmentation.) Yet, the torus derived from the $(7,2)$ torus knot is different since its $\mathrm{CH}_{0}$, given by (8.2), is non-trivial.

\section{References}

[1] F. Bourgeois, A Morse-Bott approach to Contact Homology, Ph.D. thesis, Stanford University, 2002.

[2] F. Bourgeois, Y. Eliashberg, H. Hofer, K. Wysocki and E. Zehnder, Compactness results in symplectic field theory, Geom. Topol. 7 (2003), 799-888.

[3] Y. Chekanov, Differential algebra of Legendrian links, Invent. Math. 150(3) (2002), 441-483.

[4] T. Ekholm, Morse flow trees and Legendrian contact homology in 1-jet spaces, Geom. Topol. 11 (2007), 1083-1225.

[5] T. Ekholm, J. Etnyre and M. Sullivan, Non-isotopic Legendrian submanifolds in $\mathbb{R}^{2 n+1}$, J. Diff. Geom. 71(1) (2005), 85-128.

[6] - The contact homology of Legendrian submanifolds in $\mathbb{R}^{2 n+1}$, J. Diff. Geom. 71(2) (2005), 177-305.

[7] - Orientations in Legendrian contact homology and exact Lagrangian immersions, Int. J. Math. 16(5) (2005), 453-532.

[8] - Legendrian contact homology in $P \times \mathbb{R}$, Trans. Amer. Math. Soc. 359(7) (2007), 3301-3335.

[9] T. Ekholm, K. Honda and T. Kálmán, Legendrian knots and exact Lagrangian cobordisms, in preparation.

[10] Y. Eliashberg, Invariants in contact topology, Proc. Int. Congress of Mathematicians, Vol. II, Berlin, 1998. Doc. Math. (1998), Extra Vol. II, 327-338.

[11] Y. Eliashberg, A. Givental and H. Hofer, Introduction to symplectic field theory, GAFA 2000 (Tel Aviv, 1999). Geom. Funct. Anal. 2000, Special Volume, Part II, 560-673.

[12] K. Fukaya, Y.-G. Oh, H. Ohta and K. Ono, Lagrangian intersection Floer theory anomaly and obstructon, preprint, 2000.

[13] H. Hofer, K. Wysocki and E. Zehnder, A general fredholm theory I: a splicing-based differential geometry, arXiv:math/0612604

[14] — A general fredholm theory II: implicit function theorems, arXiv:0705.1310

[15] T. Kálmán, Contact homology and one parameter families of Legendrian knots, Geom. Topol. 9 (2005), 2013-2078.

[16] L. Ng, Knot and braid invariants from contact homology. I, Geom. Topol. 9 (2005), 247-297. 
[17] - Knot and braid invariants from contact homology. II, With an appendix by the author and Siddhartha Gadgil, Geom. Topol. 9 (2005), 1603-1637.

[18] — Computable Legendrian invariants, Topology 42 (2003), 55-82.

Department of Mathematics

UPPSALA UNIVERSITY

Box 480

75106 UPPSALA, SWEDEN

E-mail address: tobias@math.uu.se

UNIVERSITY OF TOKYO

Graduate School of Mathematical Sciences

3-8-1 KOMABA

Meguro

TOKYO 153-8914, JAPAN

E-mail address: kalman@ms.u-tokyo.ac.jp

Received 10/23/2007, accepted 04/02/2008

TE is an Alfred P. Sloan Research Fellow, acknowledges support from NSF-grant DMS0505076 and from the Swedish Royal Academy of Sciences, the Knut and Alice Wallenberg foundation. TK is a research fellow of the Japan Society for the Promotion of Science. 